\title{
Review Paper \\ Medicinal Properties of Boron Supplementation on the Prevention and Treatment of Diseases: A Systematic Review
}

\author{
*Somaye Nikkhah ${ }^{1} \odot$, Mohammad Reza Naghii ${ }^{2}$
}

1. Department of Midwifery, Faculty of Nursing and Midwifery, Kerman Branch, Islamic Azad University, Kerman, Iran.

2. Department of Nutrition, School of Health, Baqiyatallah (a.s.) University of Medical Sciences, Tehran, Iran.

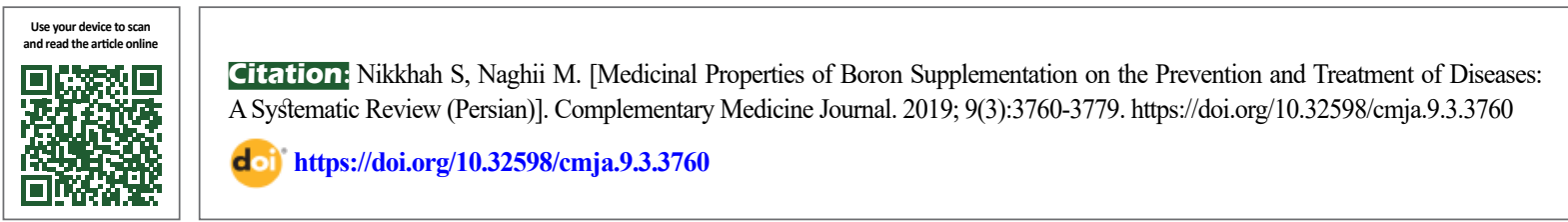

\section{(1) (8)}

Article Info:

Received: 21 Jul 2019

Accepted: 01 Sep 2019

Available Online: 01 Nov 2019

Key words:

Boron supplement Treatment, Prevention, Boric acid, Calcium fructoborate

\section{A B STRACT}

Objective Boron supplement is an essential ingredient for humans approved the American Food and Drug Administration. Since today, the use of dietary supplements for the treatment of diseases has received much attention, this systematic review study aimed to determine the effect of boron supplement on the treatment and prevention of various diseases.

Methods The search was conducted among papers published until July 2019 and indexed in Web of Science, Scopus, PubMed, Science Direct, Google Scholar, SID, IranMedex and MagIran databases using keywords of "boron supplementation", "boric acid", "calcium fructoborate", "treatment", and "prevention". Articles with a score of 3 or more based on the Jadad Scale were included in the study ( $n=67)$.

Results Fifteen preventive therapeutic effects of boron supplement were investigated. All studies confirmed the favorable effect of boron supplementation in the fields of treatment and prevention and no side effects were reported in any of them.

Conclusion Symptoms of boron deficiency such as amnesia, osteoporosis, degenerative diseases, hormonal disorders and decreased libido are prevalent, and this deficiency have many side effects that can be prevented. Hence, the use of boron as a reasonable nutritional intervention and appropriate alternative to conventional chemical drugs is recommended.

\section{Extended Abstract}

\section{Introduction}

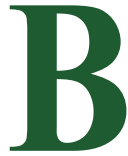

oron supplement is one of the microorganisms approved by the US Food and Drug Administration and plays an important role in people's health. Boric acid and calcium fructoborate are two boron-containing compounds. The main form of boron in body tissues is boric acid and the rest is found in the form of borate anion. Considering the regulation of plasma boron levels through renal excretion, toxicity caused by boron consumption in humans is rare and usually has no adverse effects on humans. People usually consume boron via drinking water and food intake. The daily intake of boron is about 1-3 mg/day for most adults.

The maximum recommended dose for pregnant and lactating women aged over 19 years and all adults is $20 \mathrm{mg}$ / day. The major sources of this ingredient are nuts, dried

\section{* Corresponding Author:}

Somaye Nikkhah, MSc.

Address: Department of Midwifery, Faculty of Nursing and Midwifery, Kerman Branch, Islamic Azad University, Kerman, Iran.

Tel: +98 (915) 1309039

E-mail: nikkhah_somayeh@yahoo.com 
fruits, grains, fresh vegetables, and fruits. Boron has a positive effect on the growth of bone and central nervous system, hormone regulation, reducing the risk of some types of cancer, improvement of arthritis and associated heart disease symptoms, speeding up the wound healing, pain reduction in gynecological diseases, and kidney stones through reduction of cytokines. Studies show that dietary regimes with low boron intake are common and this deficiency leads to problems in public health and increasing the risk of cancer. In this systematic review, we aimed to investigate the effect of boron supplementation on the prevention and treatment of diseases.

\section{Materials and Methods}

In this review study, we review all studies related to the preventive and therapeutic effects of boron supplementation published until July 2019 and indexed in Web of Science, Scopus, PubMed, ScienceDirect, Google Scholar, SID, IranMedex, and MagIran databases. For the search, the keywords of "Boron Supplementation", "Boric Acid", "Calcium Fructoborate", "Treatment", and "Prevention" were used. Those studies which were related to effectiveness of boron supplementation in the medical field and had a score of 3 or more based on the Jadad Scale were included in the study. Based on initial evaluation, 437 articles were found. of these, 66 papers and 7 books were reviewed. Of 66 papers, only one was in Persian and the rest were in English (Figure 1).

\section{Findings}

Boron deficiency is harmful to the human body, leading to problems in public health and an increased risk of cancer and even death. The most common symptoms of boron deficiency are arthritis, amnesia, osteoporosis, degenerative and soft cartilage diseases, hormonal disorders, and decreased libido. Studies have shown that the boron intake of less than $1 \mathrm{mg}$ per day creates a context for some problems in the human body and prevents from its benefits to the body.

In this study, of 67 reviewed articles, 15 were related to the preventive and therapeutic effects of boron including its

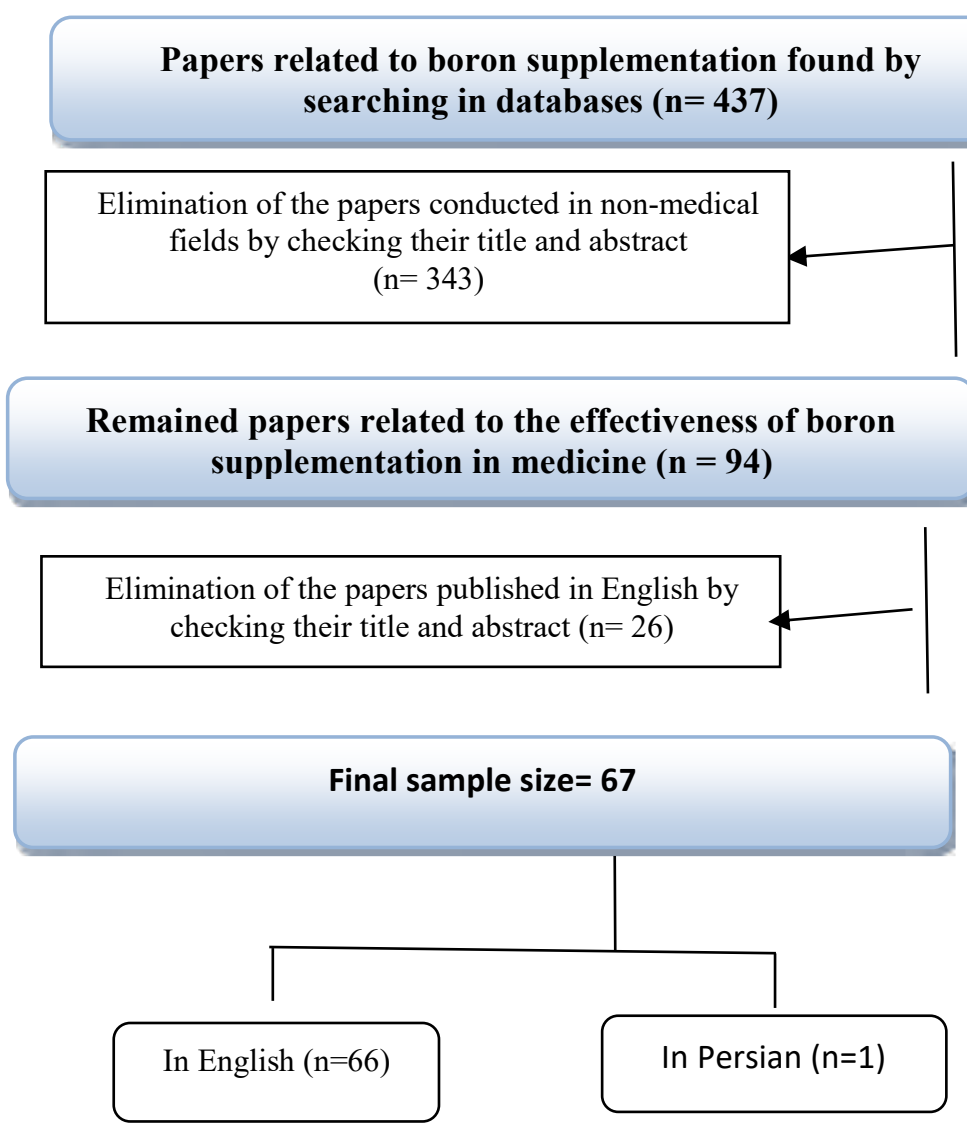

Figure 1. Flowchart of the sampling process 
Table 1. Different domains of the medicinal properties of boron supplementation in the prevention and treatment of diseases

\begin{tabular}{|c|c|c|}
\hline Number & Domain & Main Finding \\
\hline 1 & Osteoporosis & Significant effect on bone strength \\
\hline 2 & $\begin{array}{l}\text { Inflammatory } \\
\text { biomarkers }\end{array}$ & Significant reduction in inflammatory biomarkers \\
\hline 3 & CRP & Significant decrease in CRP, fibrinogen, and Erythrocyte Sedimentation Rate (ESR) \\
\hline 4 & $\begin{array}{l}\text { Cardiovascular } \\
\text { system }\end{array}$ & $\begin{array}{l}\text { Significant decrease in nitroglycerin consumption, frequency of angina attacks, and pain after the interven- } \\
\text { tion }\end{array}$ \\
\hline 5 & Pain relief & $\begin{array}{l}\text { Significant decrease in the mean severity and duration of pain in the boron supplementation group before } \\
\text { and after intervention }\end{array}$ \\
\hline \multirow{2}{*}{6} & $\begin{array}{l}\text { Kidney stones } \\
\text { in animals }\end{array}$ & Significant decrease in calcium crystal deposition in the intervention group compared to the control group \\
\hline & $\begin{array}{l}\text { Kidney stones } \\
\text { in humans }\end{array}$ & $\begin{array}{l}\text { Elimination of } 9 \times 20 \mathrm{~mm} \text { stone is associated with significant reduction in pain and reduction of hematuria } \\
\text { without hydronephrosis }\end{array}$ \\
\hline 7 & $\begin{array}{l}\text { Central ner- } \\
\text { vous system }\end{array}$ & $\begin{array}{l}\text { Significant decrease in brain magnesium and phosphorus concentration in the boron-deprived group and } \\
\text { less activity of rats in this group }\end{array}$ \\
\hline 8 & $\begin{array}{l}\text { Prostate } \\
\text { Cancer }\end{array}$ & Significant difference in prostate size between the two study groups \\
\hline 9 & $\begin{array}{l}\text { Cervical } \\
\text { Cancer }\end{array}$ & Significant difference in cervical cytopathology changes between the two groups \\
\hline 10 & Lung cancer & Significant reduction of lung cancer incidence between the two study groups \\
\hline 11 & Breast cancer & Significant reduction of genotoxic changes in the medium containing boric acid \\
\hline 12 & Liver cancer & $\begin{array}{c}\text { Significant decrease in Proliferating Cell Nuclear Antigen (PCNA) and as a result, significant decrease in liver } \\
\text { cancer }\end{array}$ \\
\hline 13 & Melanoma & Induction of apoptosis in melanoma cells in vitro \\
\hline 14 & Hormones & significant increase in mean plasma concentration of free testosterone and a significant decrease in estradiol \\
\hline 15 & Infertility & $\begin{array}{l}\text { Modification of the abnormal form of sperm in one man and improvement of sperm motility in another } \\
\text { person and increasing sperm count in both cases }\end{array}$ \\
\hline
\end{tabular}

effect on osteogenesis, inflammatory response, C-Reactive Protein (CRP), cardiovascular system, pain relief, kidney stone, central nervous system, prostate cancer, cervical cancer, lung cancer, breast cancer, liver cancer, melanoma, hormones, and infertility (Table 1). The use of boron in pharmaceuticals is also on the rise.

\section{Conclusion}

Boron has a positive effect on the growth of bone and central nervous system, hormone regulation, reducing the risk of some types of cancer, improvement of arthritis and associated heart disease symptoms, speeding up the wound healing, pain reduction in gynecological diseases, and kidney stones. Despite the need for boron intake of 1-3 mg per day in adults, symptoms of boron deficiency such as arthritis, amnesia, osteoporosis, degenerative and soft carti- lage diseases, hormonal disorders, and decreased libido are still common, and this deficiency can have many adverse effects that can be prevented. Therefore, its consumption as a reasonable dietary and a suitable alternative for common chemical drugs is recommended.

\section{Ethical Considerations}

\section{Funding}

This research did not receive any specific grant from funding agencies in the public, commercial, or not-forprofit sectors.

\section{Authors' contributions}

Conceptualization, investigation, analysis and resources, writing-review \& editing by all authors; initial draft prepara- 
tion, visualization, supervision, project administration, funding acquisition: Somaye Nikkhah.

Conflicts of interest

The authors declared no conflict of interest.

Acknowledgment

The authors would like to thank Dr. Nielsen and Dr. Hunt for their valuable cooperation. 
This Page Intentionally Left Blank 


\title{
خواص دارويى مكمل بورون در ييشتَيرى و درمان بيمارىها: مقاله مرورى منظم
}

\author{
"سميه نيكخواه' م، محمدرضا نقى \\ ا. I. كروه مامايى، دانشكده يرستارى مامايي، واحد كرمان، دانشكاه آزاداد اسلامى، كرمان، ايران.

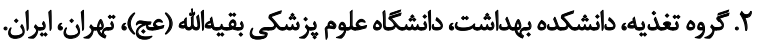

\begin{abstract}
جيكيد

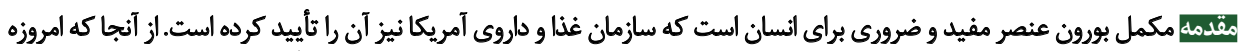

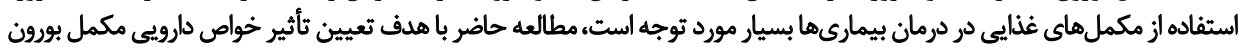

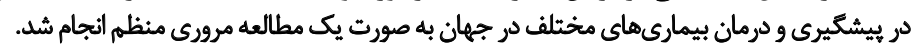

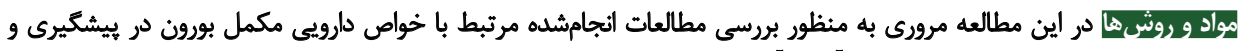

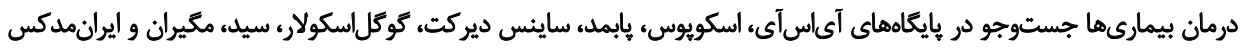

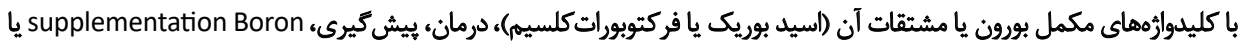

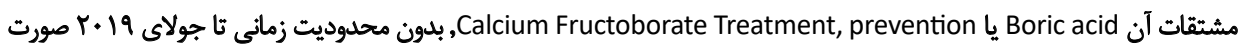

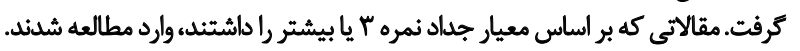

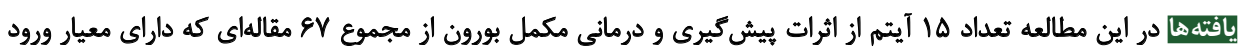

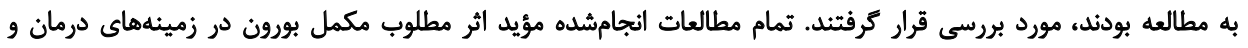

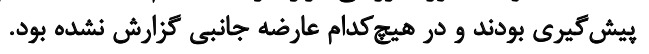

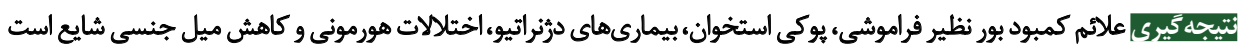

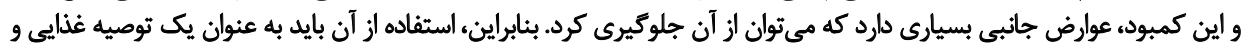

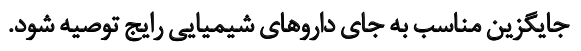

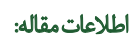

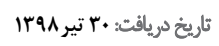

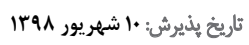

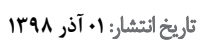

كليدوازٔهها:

مكمل بورون، درمان، فيشكيرى، اسيد بوريك، فركتوبوراتكلسيم بوريم

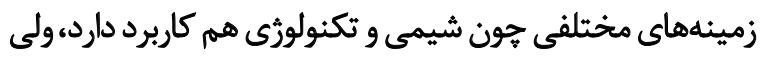

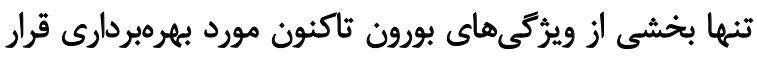

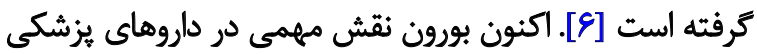

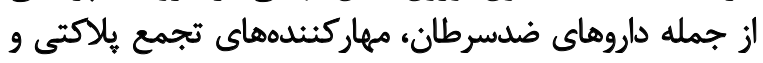

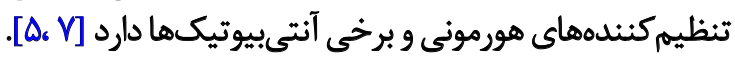

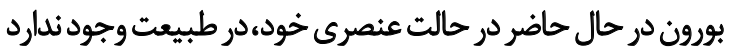

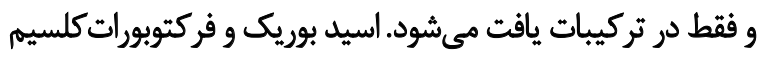

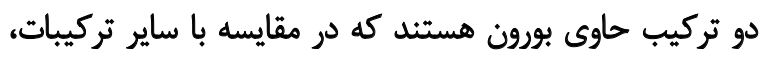

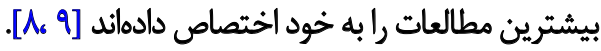

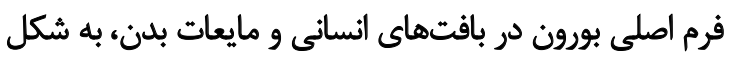

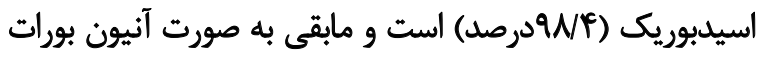

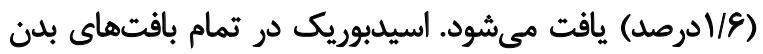

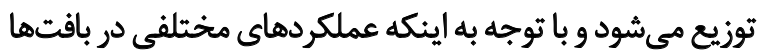

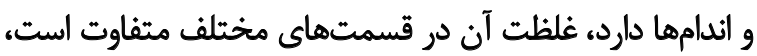

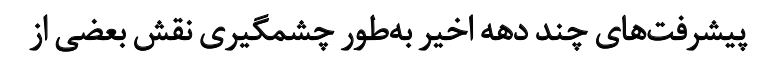

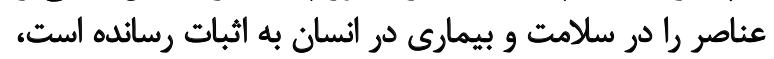

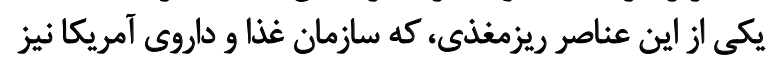

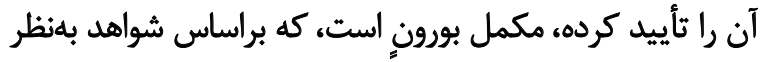

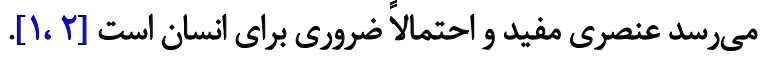

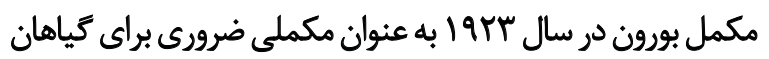

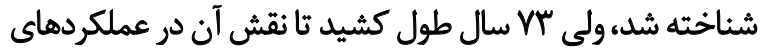

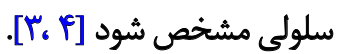

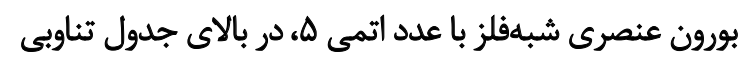

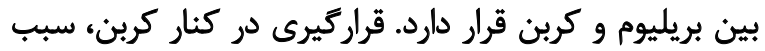

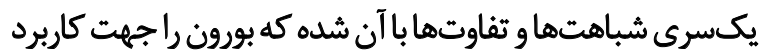

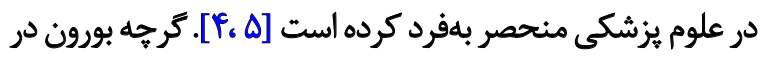

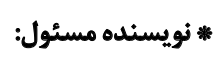
سميه نيكخواه نشائى: كرمان، دانشكاه آزاد اسلامى، واحد كرمان، دانشكده هيرستارى مامايى، كروه مامايى.

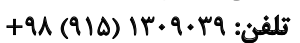
يست الكترونيكي: nikkhah_somayeh@yahoo.com 
ييشكيرى و درمانى مكمل بورون محدود به جستوجوى اينترنتى إيكي

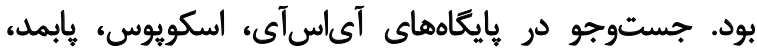

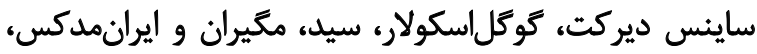

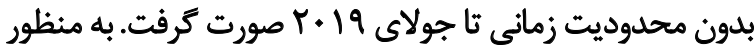

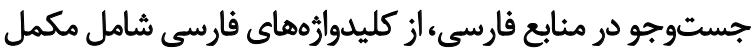

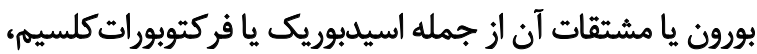

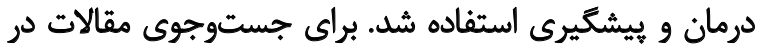

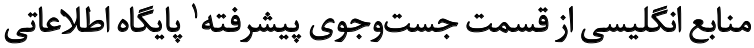

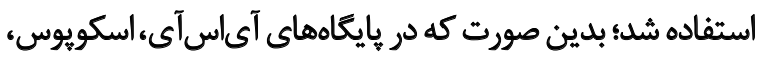

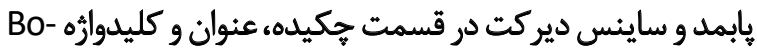

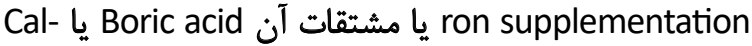
Alum Fructoborate

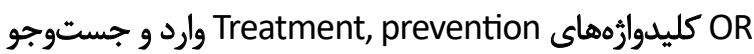

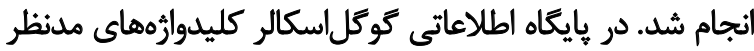

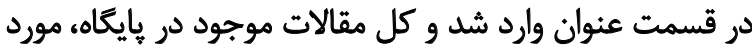
بروسى قرار ترفت.

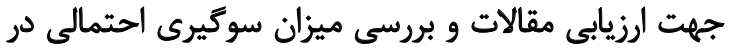

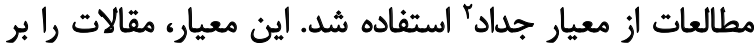

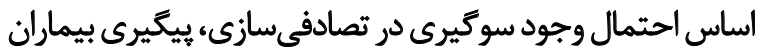

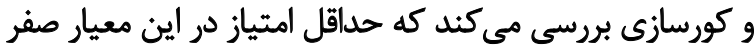

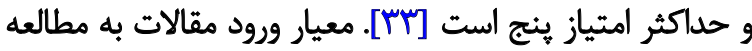

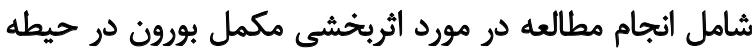

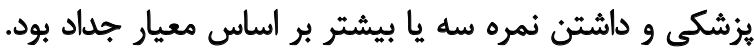

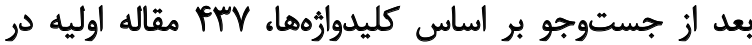

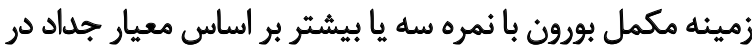

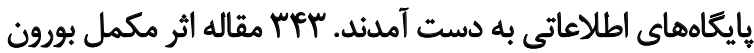

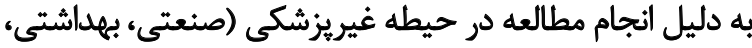

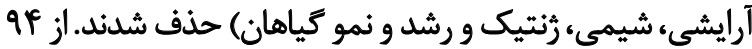

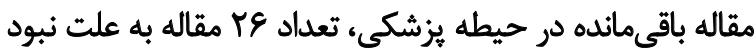

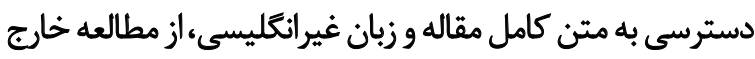

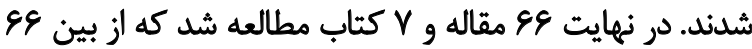

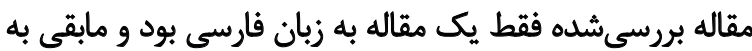

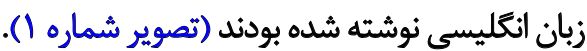

يافتّها

كمبود بورون براى بلن مضر است و در انسان به بروز مشكلاتى

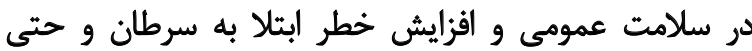

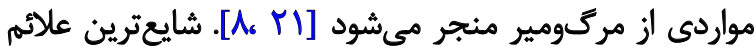

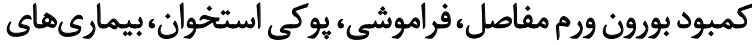

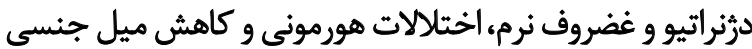

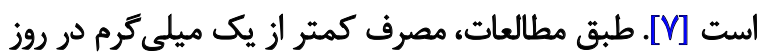

1. Advance search
بهكونهاي كه استخوان، ناخن و مو بيشترين غلظت اسيد بوريك

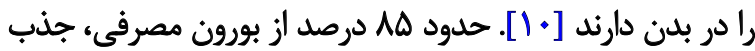

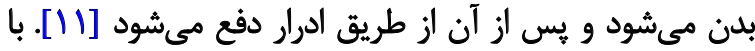

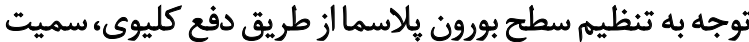

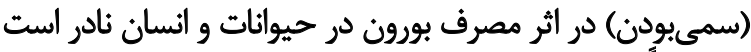

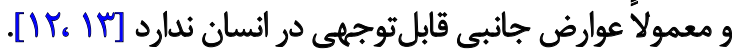
مردم بهطور مداوم بورون را از آب آشاميدنى و مواد غذايى

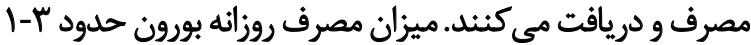

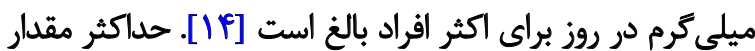

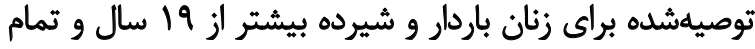

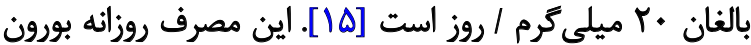

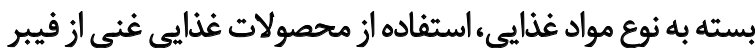

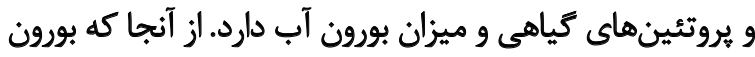

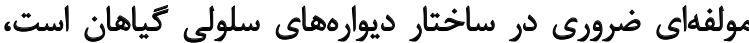

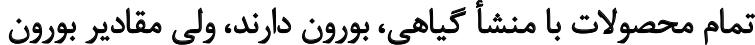

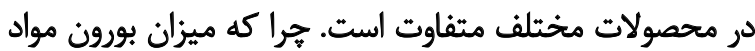

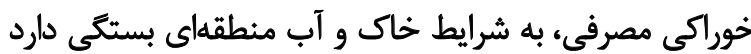

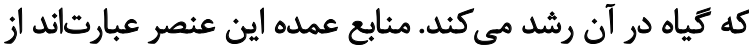

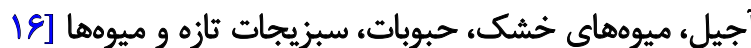

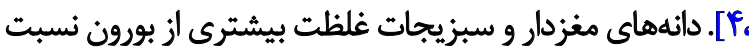

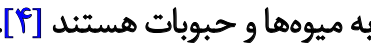

انسان و حداقل برخى از حيوانات بيشرفتهتر، به بورون براى

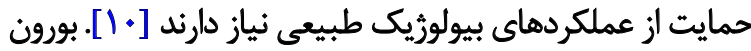

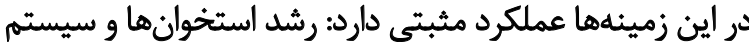

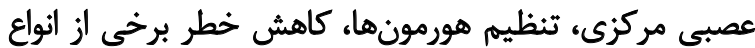

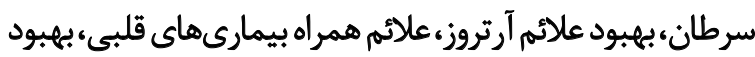

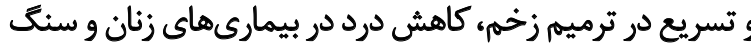

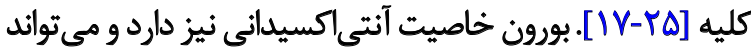

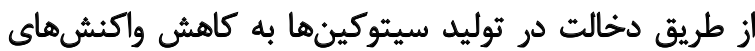

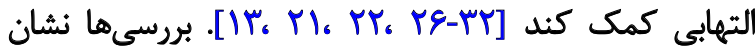
مي دهد رزيمهاى غذايى با مصرف بورون كمه، شايع است و اين

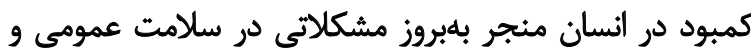

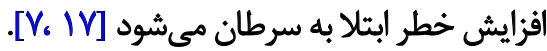

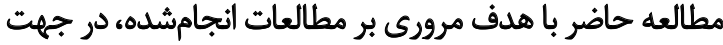

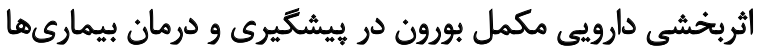

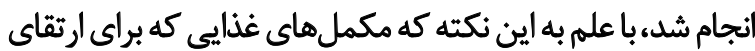

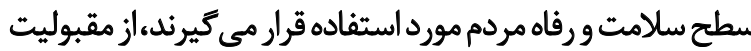
بيشترى در جامعه برخوردارند و عوارض جانبي كمترى دارند.

$$
\text { مواد و روشُ هـا }
$$

استر اتثى جستوجوى مقالات در اين مطالعه مرورى، بررسى تمامى مطالعات مرتبط با اثرات 
مقالات اوليه مكمل بورون در يايكاههاى جستجو (تعداد= FrV)

حذف مقالات با بررسى عنوان و جكيده ( تعداد= به دليل انجام مطالعه در حيطهى غير يزشكى(صنعتى، بهداشتى، آرايشى، شيمى،رنتيك و اثر آن در رشد و نمو حياهان)

مقالات باقى مانده با هدف اثربخشى مكمل بورون در هزشكى (تعداد= qF)

حذف مقالات با بررسى عنوان و جكيده ( تعداد=\&ץ) بهلت زبان غيرانكليسي در نهايت \&V مقاله وارد مطالعه شدند.

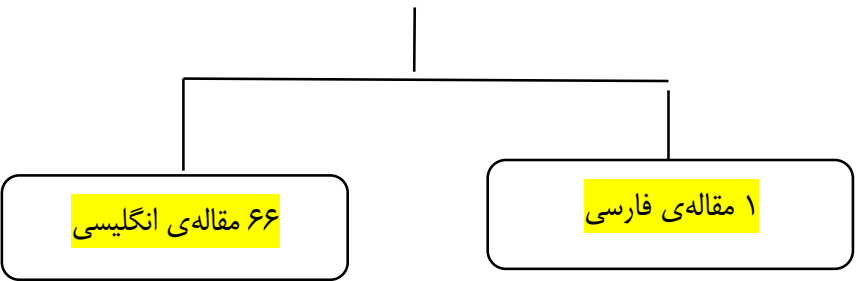

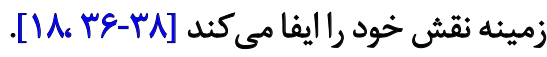

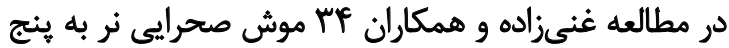

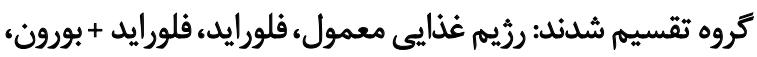

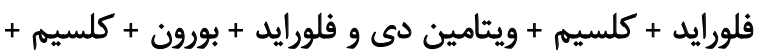

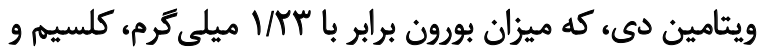

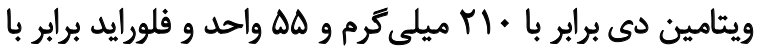

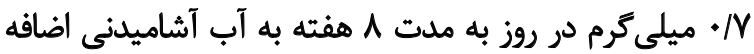

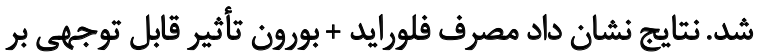

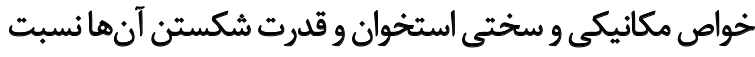
به مصرف مكمل كلسيم +ويتامين دى دارد [ [ج"] محروميت از بورون در موش، سبب كاهش ضخامت ترابكولار،

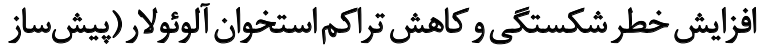

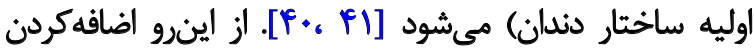

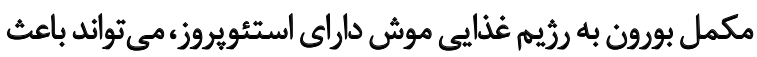

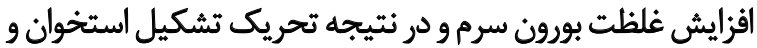

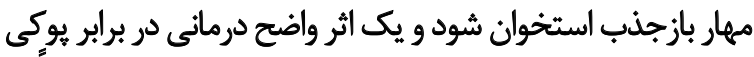

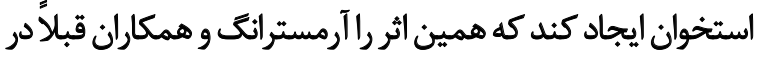

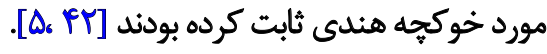

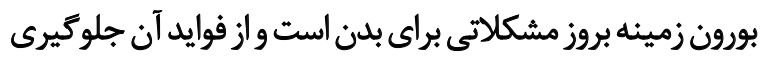

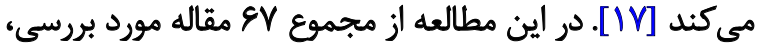

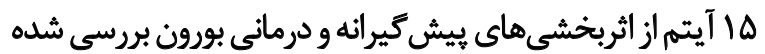

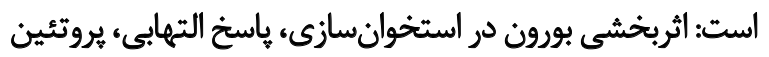

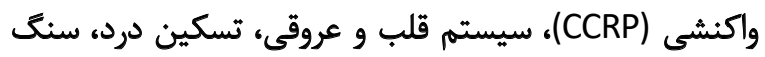

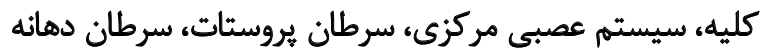

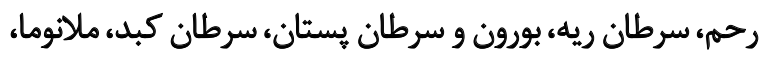
هورمونها و نابارورى (جدول شرون شماره (1).

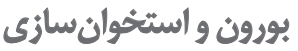

بورون از طريق تأثير بر استئوبلاست (سلول هاى مسئول تشكيل

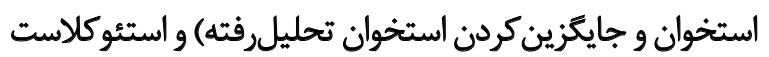

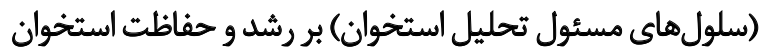

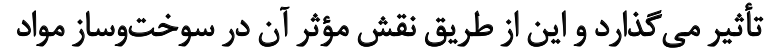

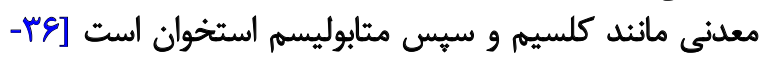

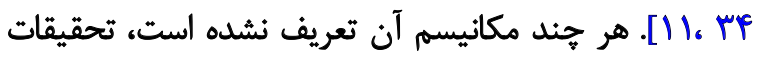

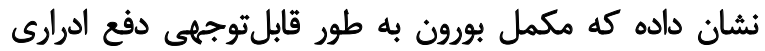

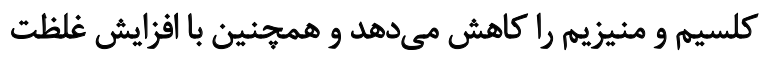

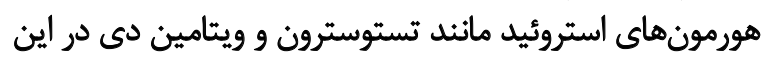


همجنين در مطالعه ريز-آيزكوايردو و همكاران بر روى · ب بيمار

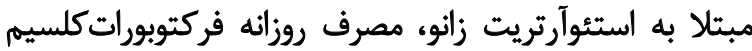

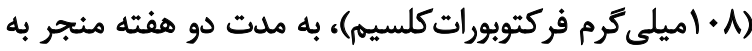

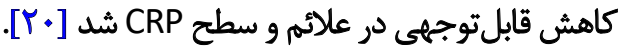

$$
\text { بورون و سيستم قلب و عروقى }
$$

افزايش سطح CRP و كلسترول HDL در خون عامل خطرى

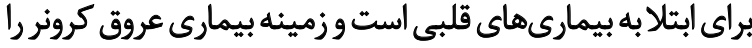

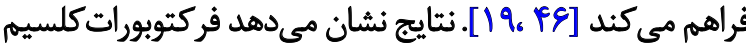
باعث كاهش قابلتوجهى در CRP و كلسترول HDL مئش

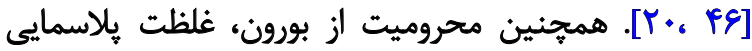

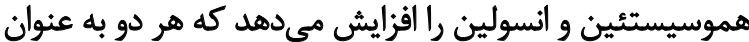

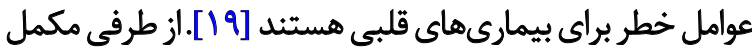

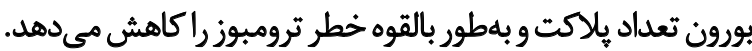

به احتمال فراوان بورون حاوى مهاركنتده ترومبين است [ـ [ب].

در مطالعه ميليتارو و همكاران بر روى افراد مبتلا به آنرئين

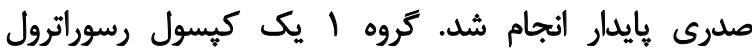

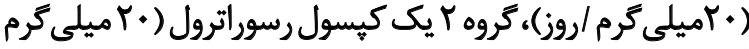

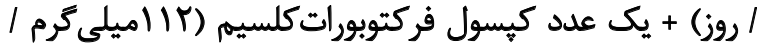

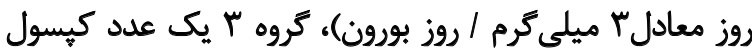

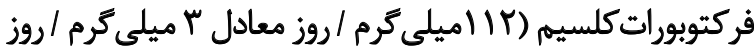

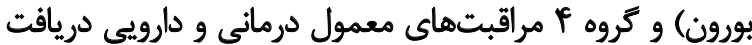

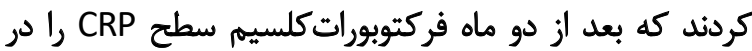

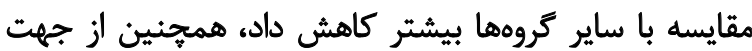

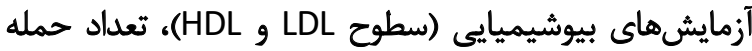

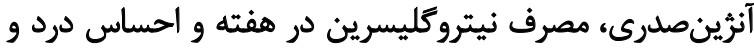

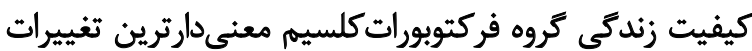

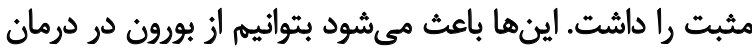
اختلالات قلبىعروقى استفاده كنيم [19].

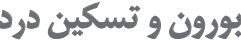

مكمل بورون در بيمارىهاى مختلفى سبب تسكين درد

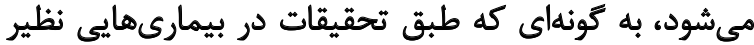

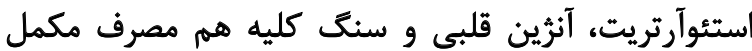

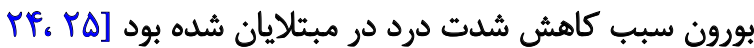

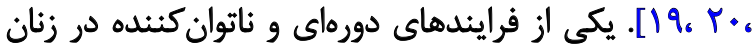

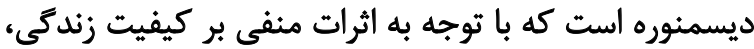

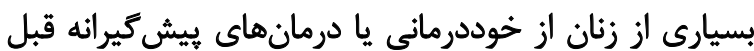

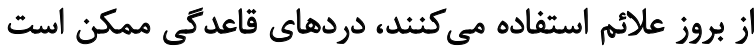

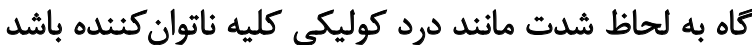
ن] [FV، FN]

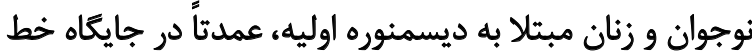
اول درمان محسوب مىشوند، اما شكست درمان بان با اين داروها

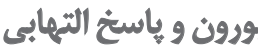

تحقيقات نشان داده كه بورون ممكن است التهاب و يا ياسخ

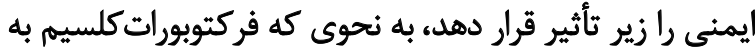

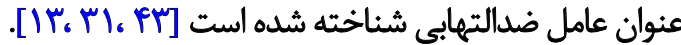
برخى از دادهاي تجربى نشان مى دهد بورون مشابه اسيدهاى

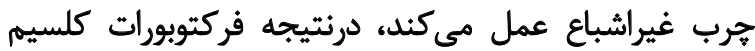

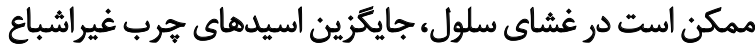

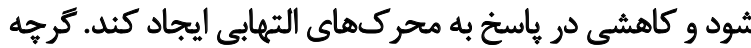

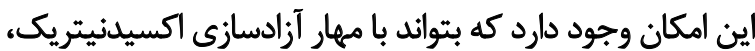

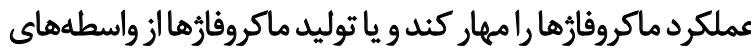

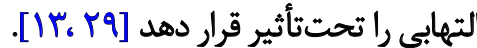

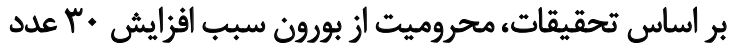

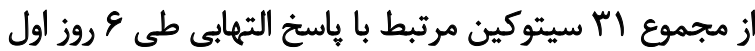

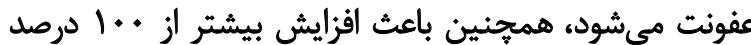

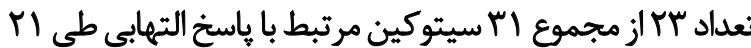
روز اول عفونت مىشود [آست.

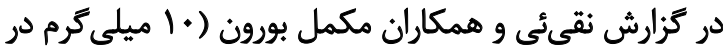

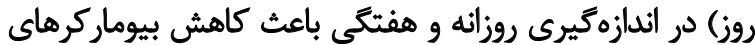

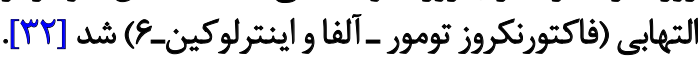
در مطالعه تجربى ديكرى، اضافهردن بورون بهرئ به رئيم غذايى

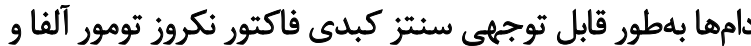

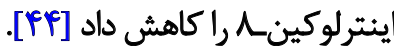

همجنين به دنبال تزريق آنتىرن مايكوباكتريوم بوتيريكوم

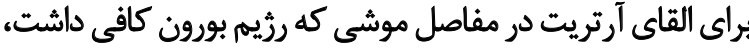

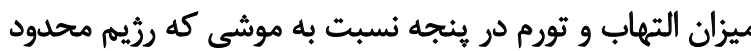

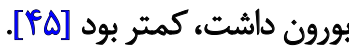

سيتوكينها مثل اينترلوكين-ا، اينترلوكين_و و و فاكتور

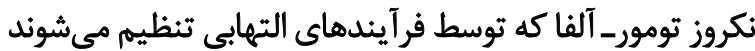

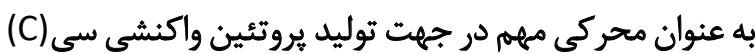

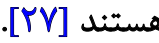

\section{بورون و هيروتئين واكتشيى"}

تا حد زيادى بين غلظت CRP و و بيشرفت بيمارى عروق

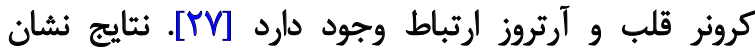

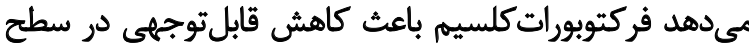

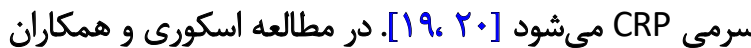

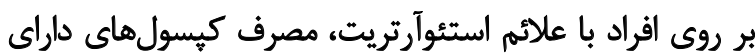

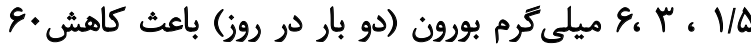

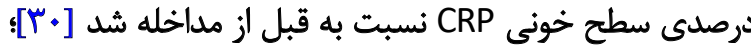

3. C-Reactive Protein (C-CRP) 
بورون در عملكردهاى شناختى نقشى مهمر ايفا مى كند،

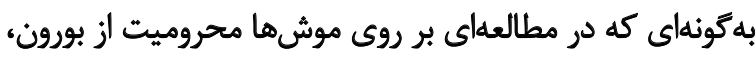

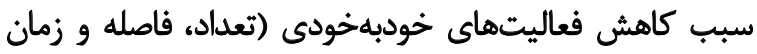

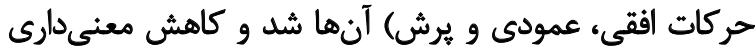

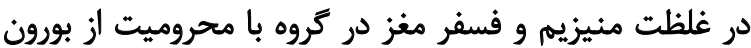

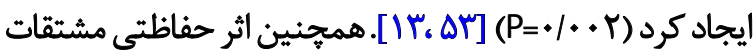

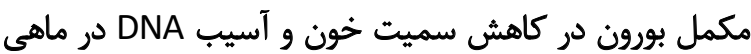

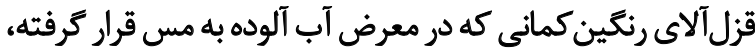

نشان داده شده است [QF].

بورون و سرطان

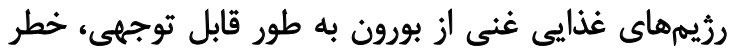

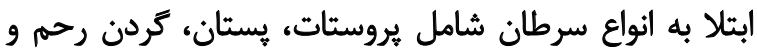

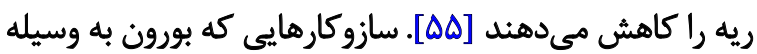

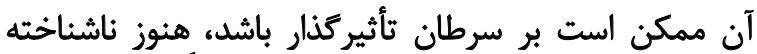

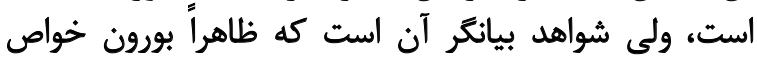

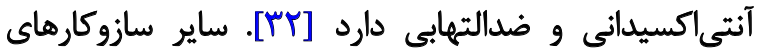

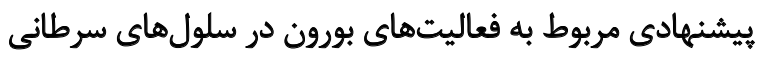

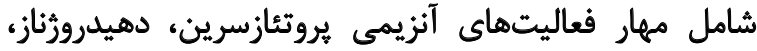

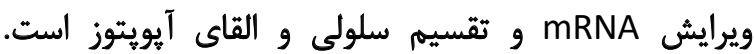

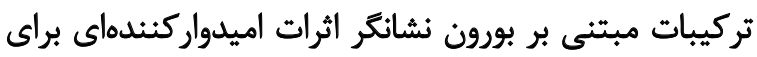
شيمي درماني انواع سرطان هان هستئد [هدان.

\section{يورون و سرطان يروسثاث}

افزايش ميزان مصرف بورون در غذا يا آب، خطر سرطان

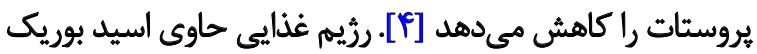

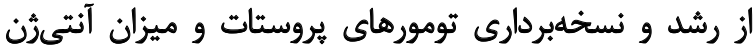

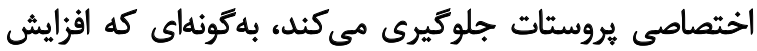

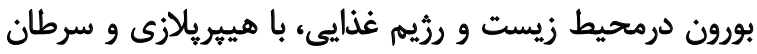

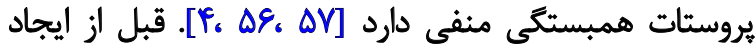

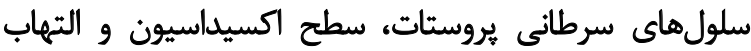

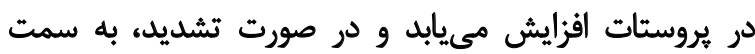

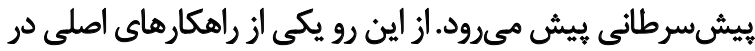

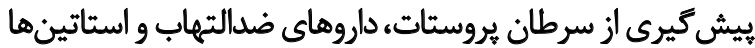

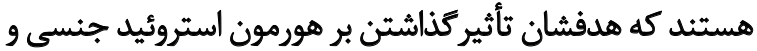

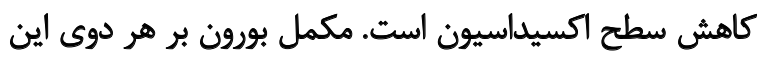
سازوكارها، مؤثر است [هر]

بزر كَشدن يروستات عامل خطر مهمى براى سرطان يروستات

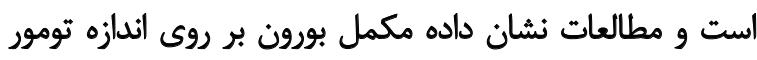

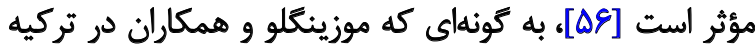

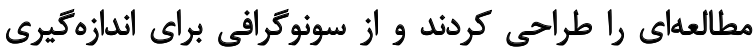

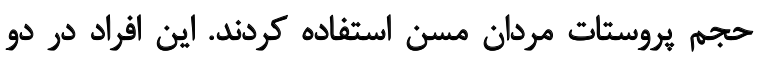

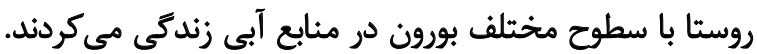

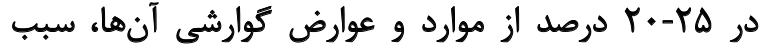

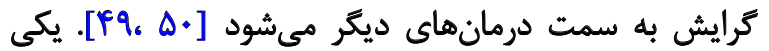

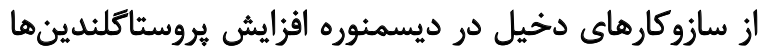

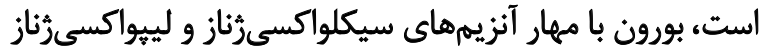

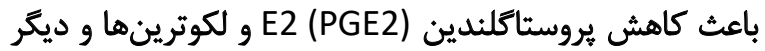

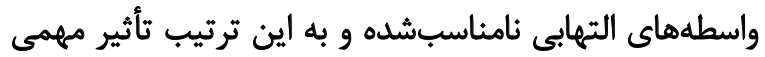

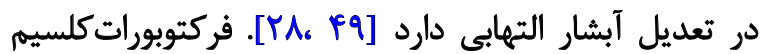

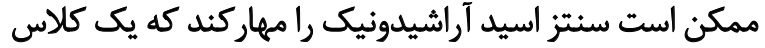

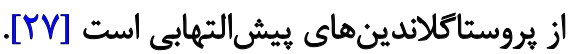

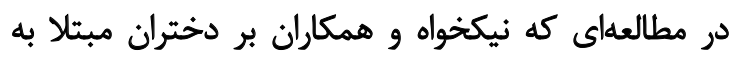

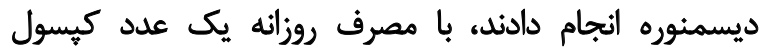

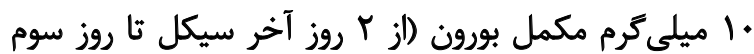

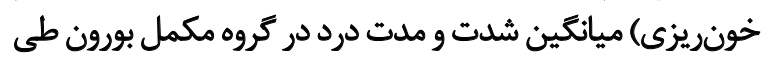

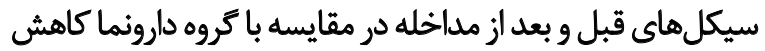

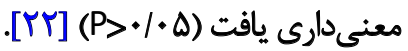

بورون و سنى كليه

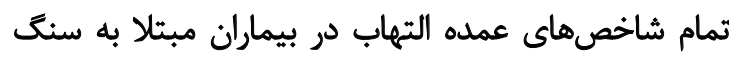

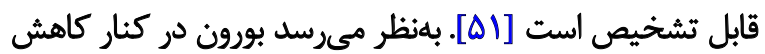

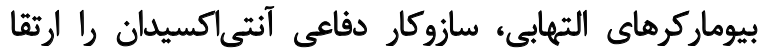

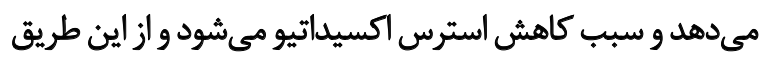

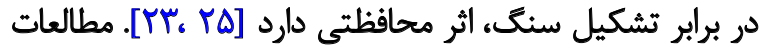

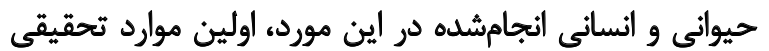

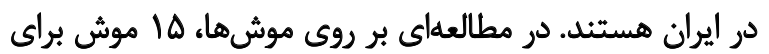

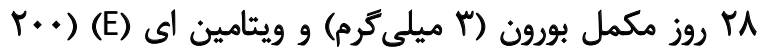

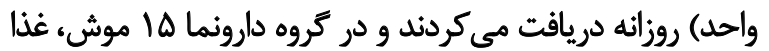

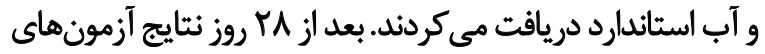

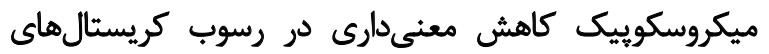

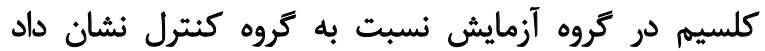

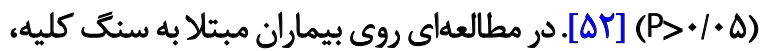

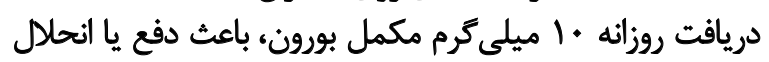

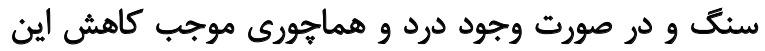

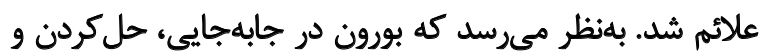

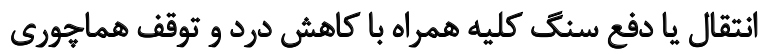

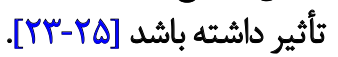

بورون و سيستم عصبى مركزى

يافتهها نشان مىدهد رزيم غذايى داراى بورون فوايدى براى دري

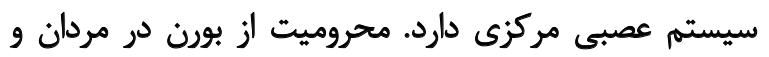

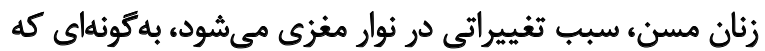

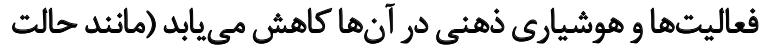

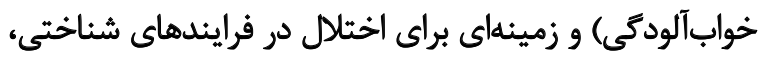
مهارتها و حافظه به وجود مئى آورد [11] 
سميت سلولى آن در سلولهاى سالم انسان موجود است. در

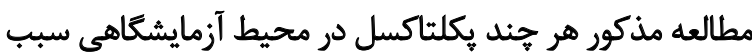

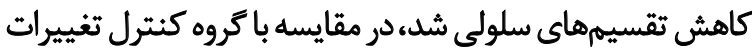

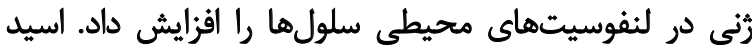

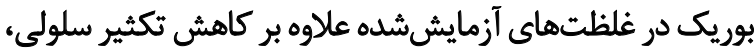

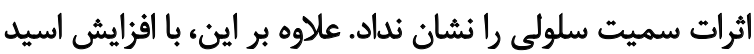

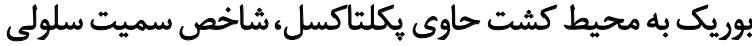
و اثرات رنوتوكسيك كاهش يافته بود [FF].

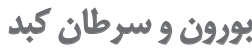

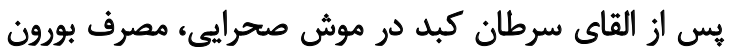

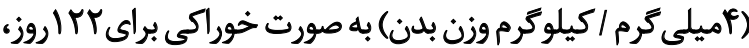

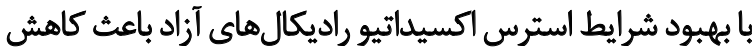

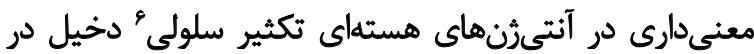

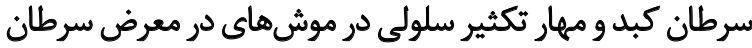

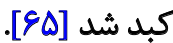

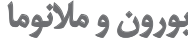

در مورد سلول هاى ملاتوم، اسيد بوريك احتمالاً تكثير رونويسى

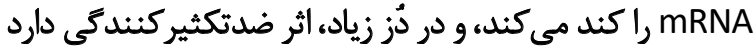

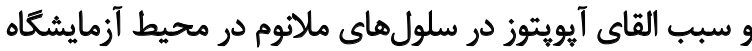

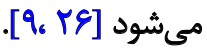

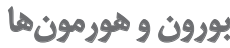

بورون نقش بيولوريكي و تنظيم كنيدكى در توليد هورمونهاي

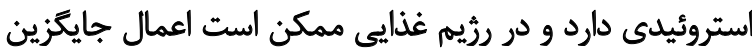

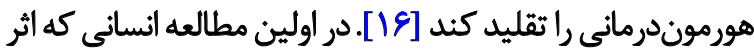

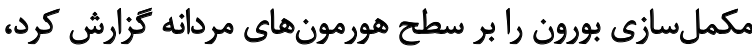

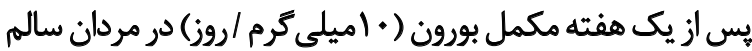

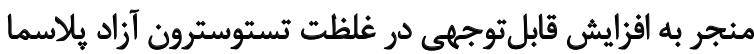

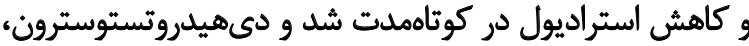

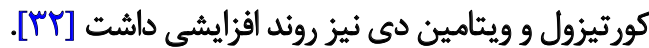

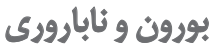

اثر افزايشدهنده تستوسترون با مصرف بورون، يكى از بازي

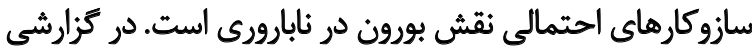

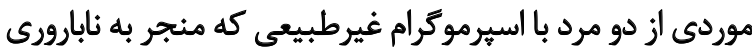

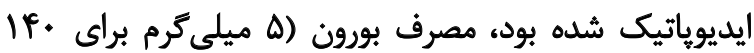

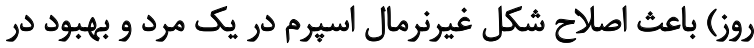

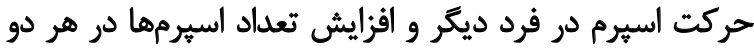

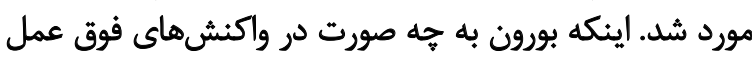

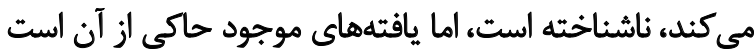

6. Proliferating Cell Nuclear Antigen (PCNA)
مردانى كه در روستاى با بورون بيشتر زندخى مي كردند، بهاطور

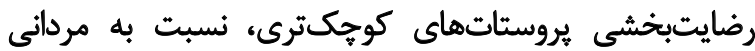

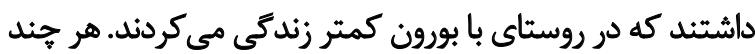

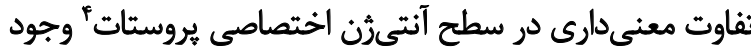

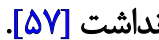

$$
\text { بورون و سرطان دهانه رحم }
$$

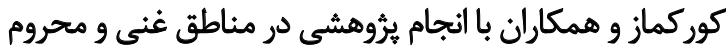

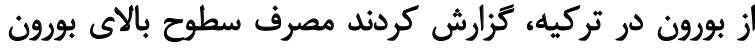

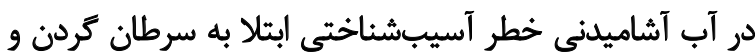

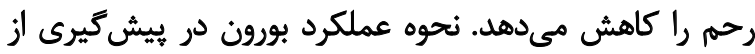

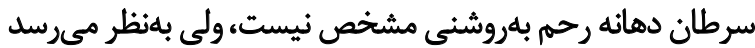

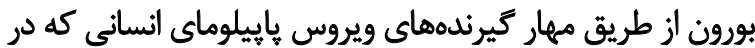

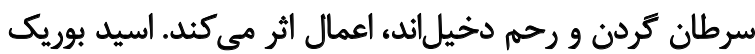

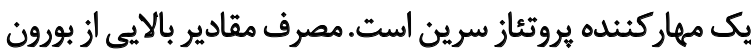

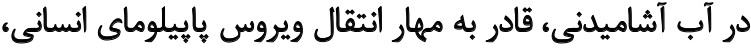

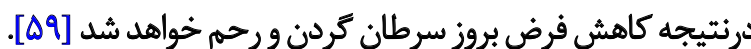

$$
\text { بورون و سرطان ريه }
$$

در مطالعه مهابير و همكاران در زنان مبتلا به سرطان ريه،

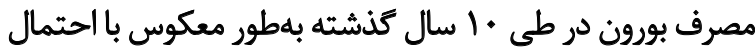
ابتلا به سرطان ريه همراه بود [• بع].

$$
\text { بون و سر طان يسثان }
$$

كلسىتريول اثر حفاظتي در برابر سرطان پريستان دارد.

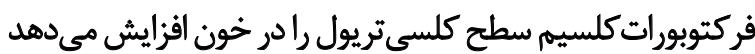

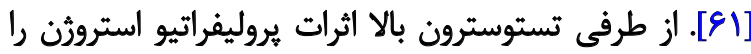

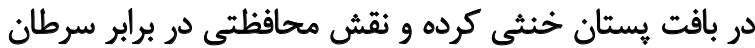

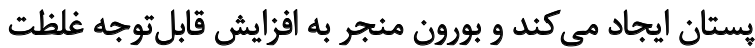

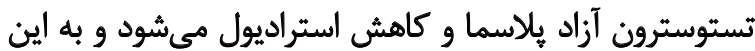

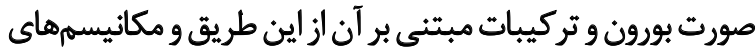

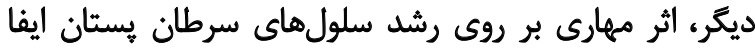

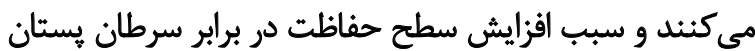

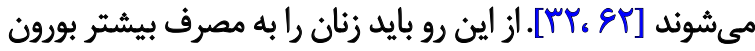
به منظور كاهش شانس ابتلا به سرطان تشويق كرد [ [عانـان. تحقيقات نشاندهنده اثر مثبت داروهاى مبتنى بر بورون در

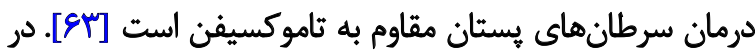

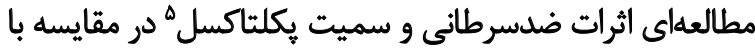

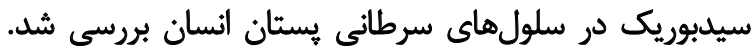

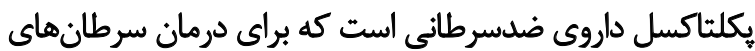

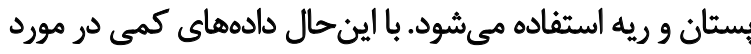

4. Prostate-Specific Antigen (PSA)

5. Paclitaxel (PAC) 


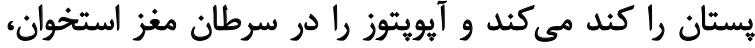

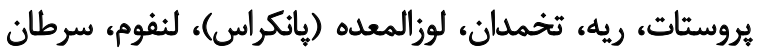

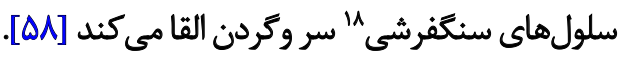

ث

مطالعه حاضر با هدف ارزيابى كارايى مكمل بورون در حيطه

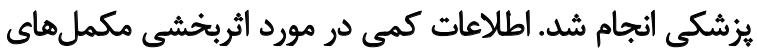

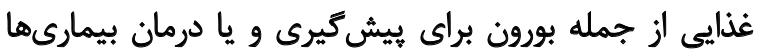

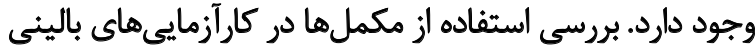

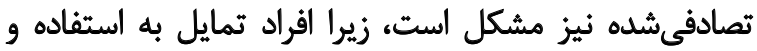

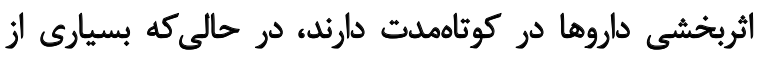

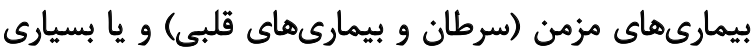

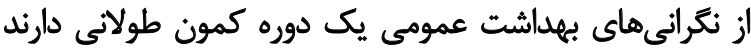

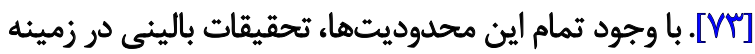

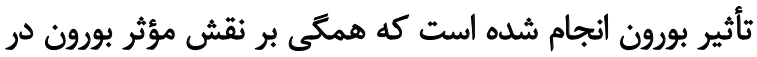
زمينههاى مختلف بيش كيرى و درمان صحه مي كذارئد

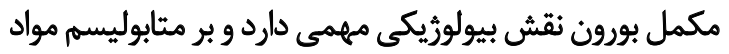

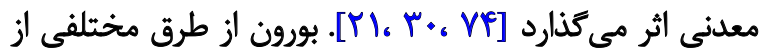

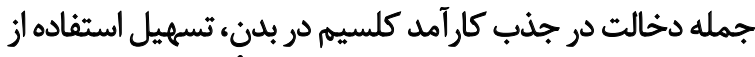

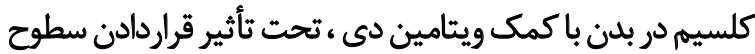

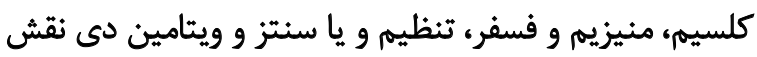
خود را در سوختوساز و كلسيفيكاسيون استخوان ايفا مئين وكند

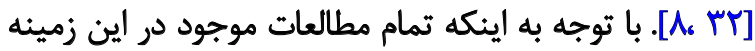

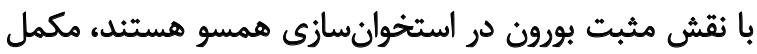

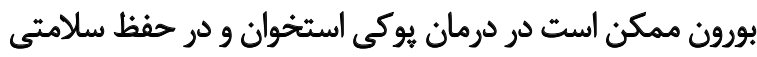

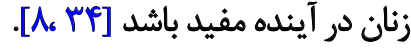

مطالعات انجامشده شواهدى رادر حمايت از اين ديد

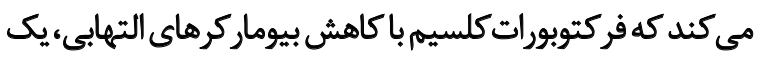

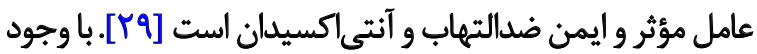

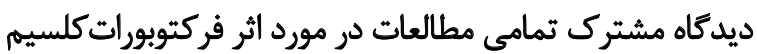

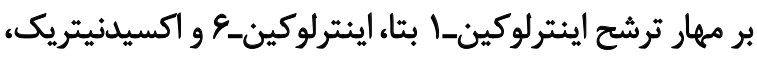

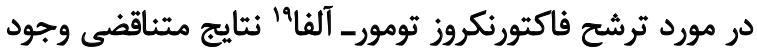

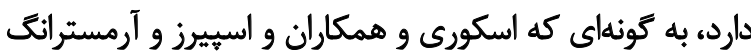

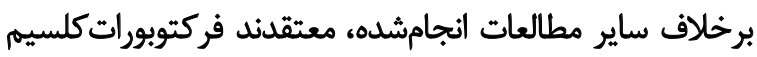

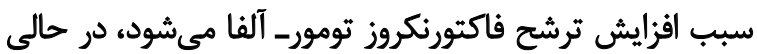

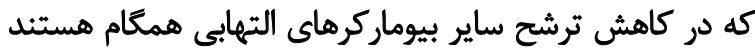

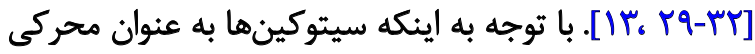

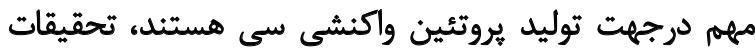

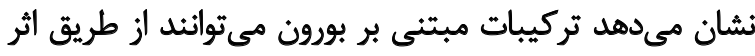

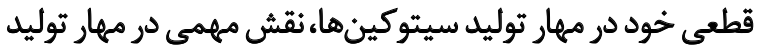

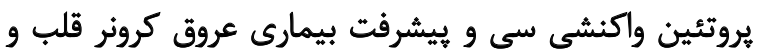

18. Squamous Cell Carcinoma (SCC)

19. Tumor Necrosis Factor alpha(TNF- $\alpha) \alpha$
كه اين عنصر بر تشكيل فرمهاى فعال يا هيدوركسيله بعضى إنتي

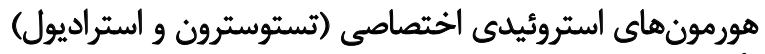

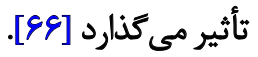

كمبود بورون در قورباغه نر سبب تحليل بيضهها، كاهش

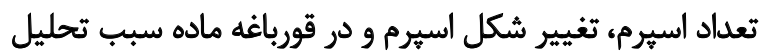

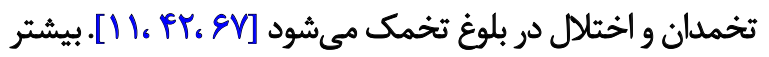

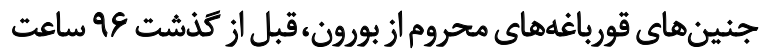

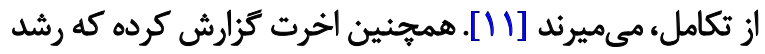

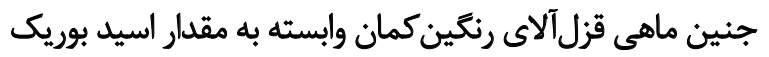

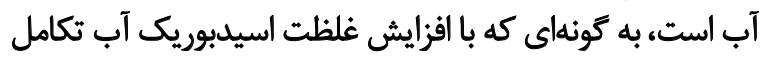

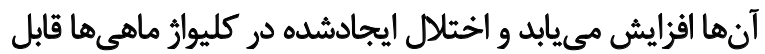

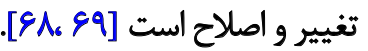

\section{بورون و الستفاده دارويى}

كاربردهاى متنوع بورون اين ايده كلى را تقويت مي كند كهي

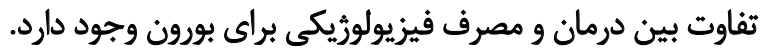

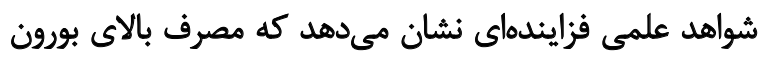

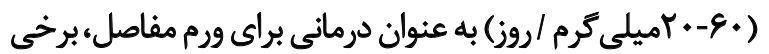

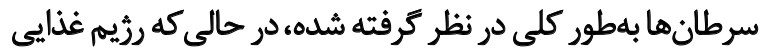

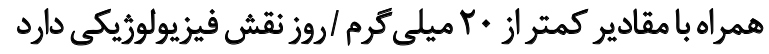

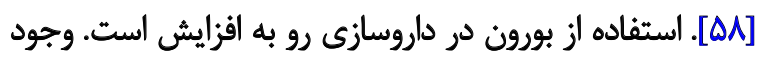

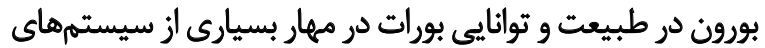

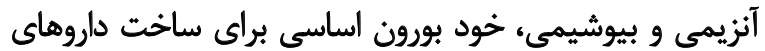

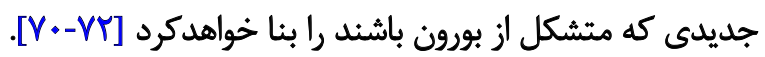

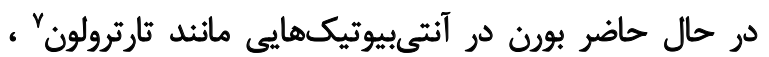

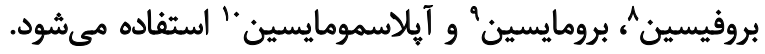

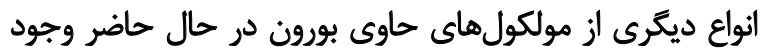

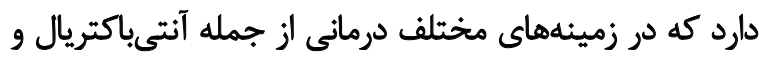

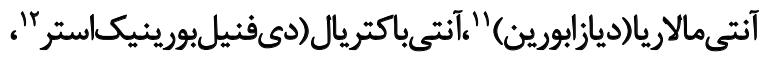

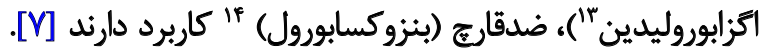

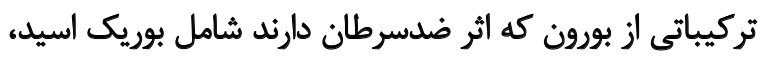

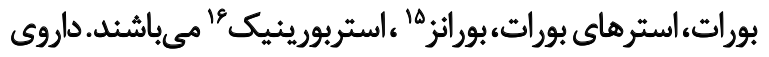
بورتزوميب"، از استرهاى بورات است كه رشد سلولهائى سرطان

\section{Tartrolon}

8. Borophycin

9. Boromycin

10. Aplasmomycin

11. Diazaborine

12. Diphenyl borinic esters

13. Oxazaborolidines

14. Benzoxaborole

15. Boranes

16. Borinic esters

17. Bortezomib 


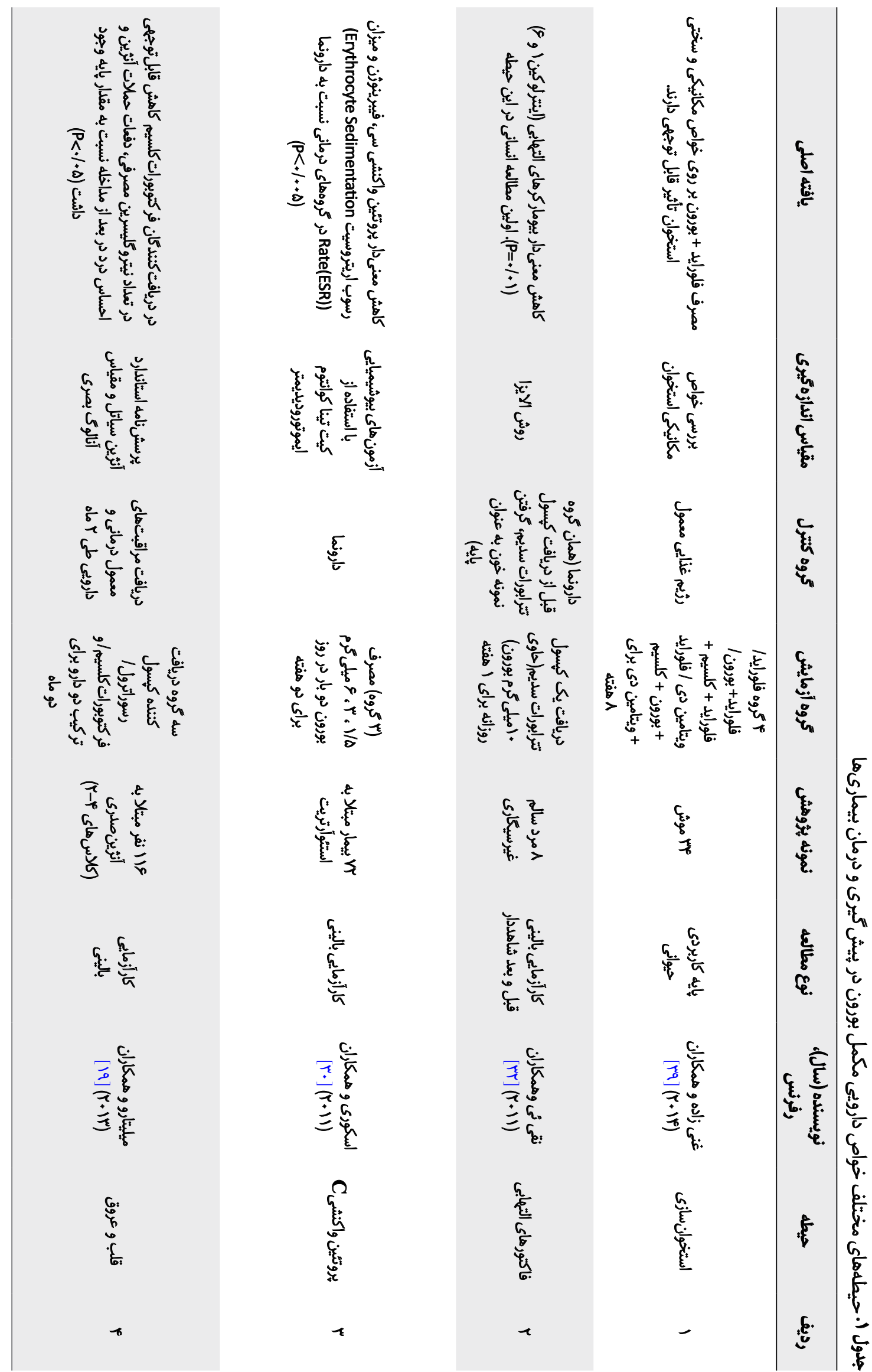




\begin{tabular}{|c|c|c|c|c|c|c|c|}
\hline 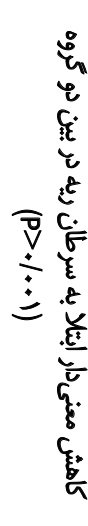 & 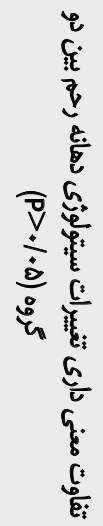 & 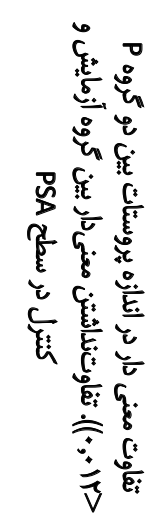 & 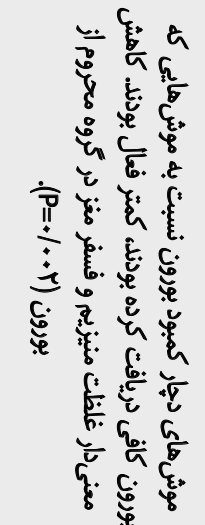 & 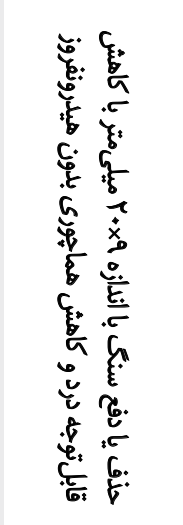 & 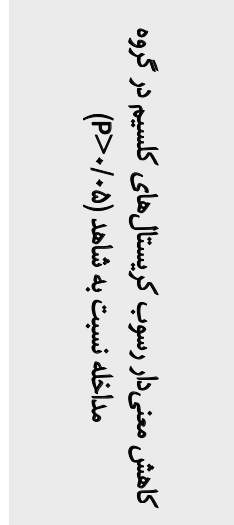 & 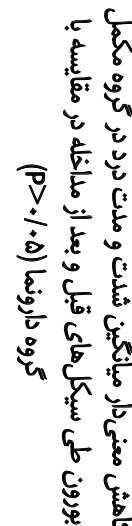 & $\frac{\mathfrak{s}}{E_{t}}$ \\
\hline 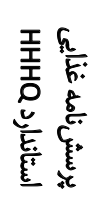 & $\frac{E}{E}$ & 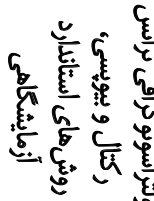 & 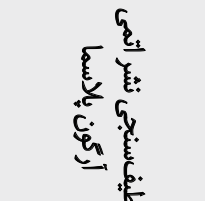 & 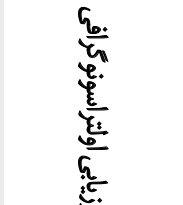 & 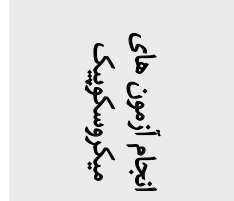 & 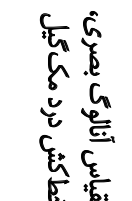 & 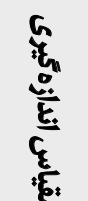 \\
\hline$\frac{z}{\underline{\varepsilon}}$ & 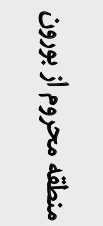 & 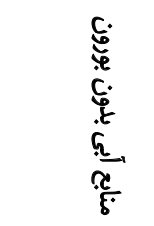 & 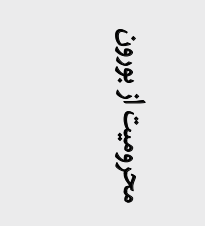 & & 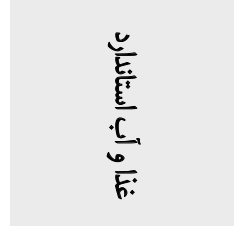 & .్ & 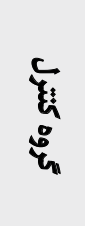 \\
\hline 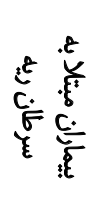 & 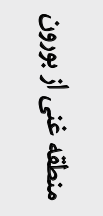 & 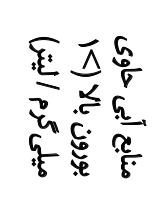 & 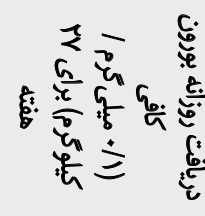 & 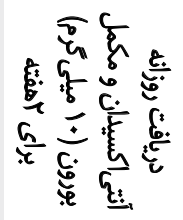 & 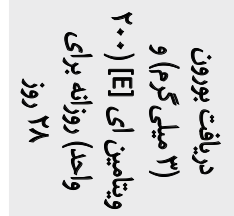 & 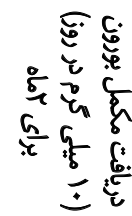 & है \\
\hline$\frac{\xi}{\tilde{r}}$ & $\frac{\mathrm{c}}{\mathrm{c}}$ & 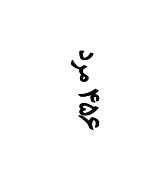 & $\begin{array}{l}\text { E. } \\
\text { \& } \\
g\end{array}$ & 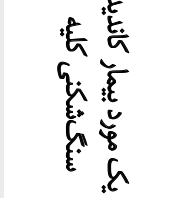 & 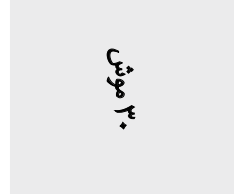 & 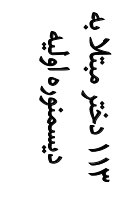 & 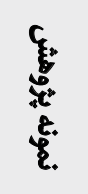 \\
\hline 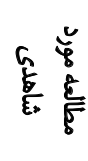 & 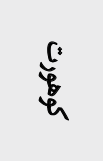 & 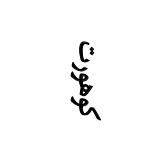 & 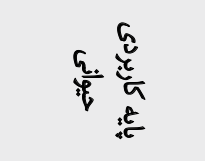 & 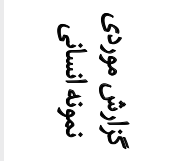 & 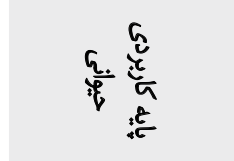 & 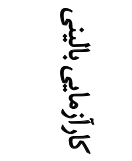 & $\begin{array}{c}\xi \\
E \\
E\end{array}$ \\
\hline 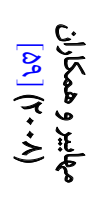 & 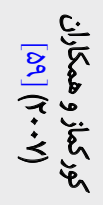 & 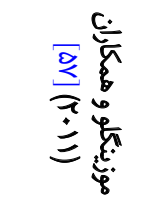 & 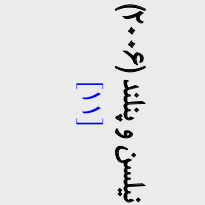 & 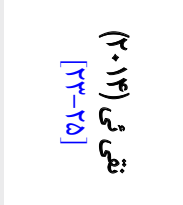 & 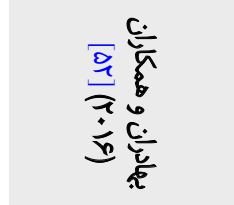 & 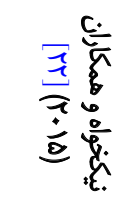 & $\frac{\hat{c}}{\frac{E}{0}}$ \\
\hline $\begin{array}{l}\varepsilon \\
\varepsilon \\
\tilde{c} \\
\xi \xi\end{array}$ & 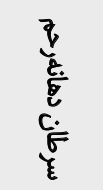 & 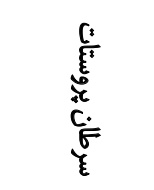 & दू है & 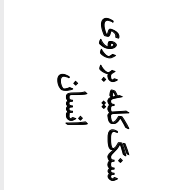 & 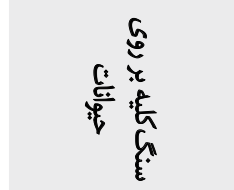 & है. & 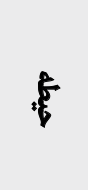 \\
\hline$\tau$ & 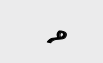 & $>$ & $<$ & $r$ & & $\theta$ & $\underbrace{E}_{0}$ \\
\hline
\end{tabular}



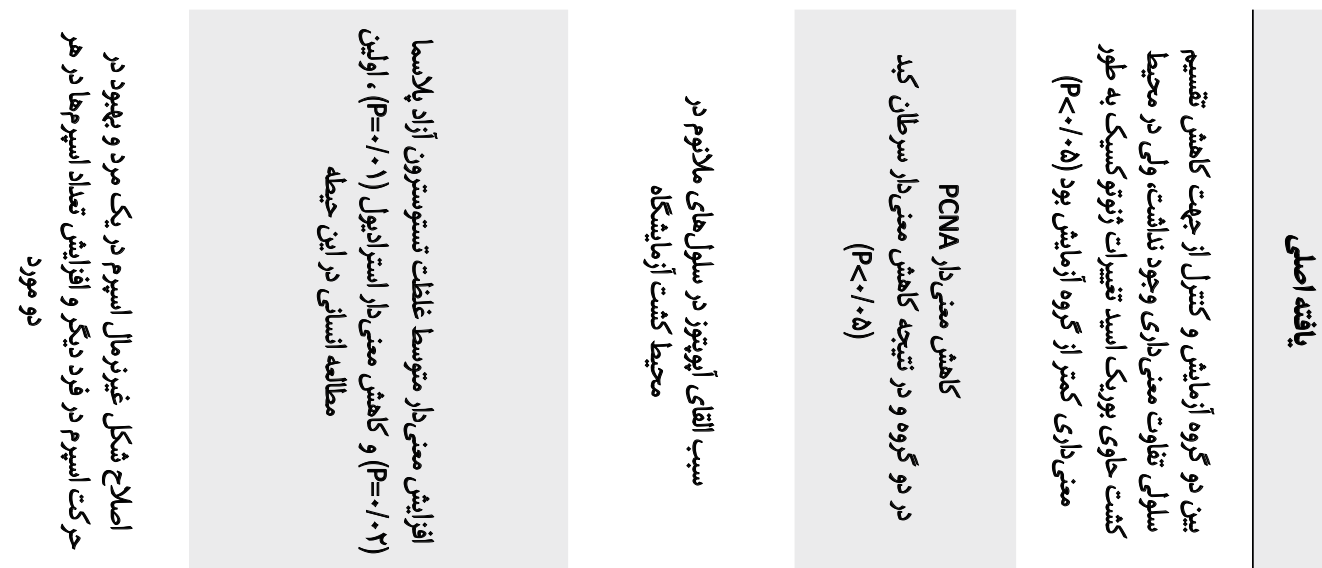

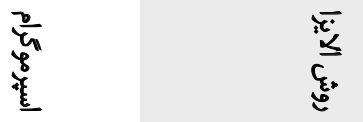

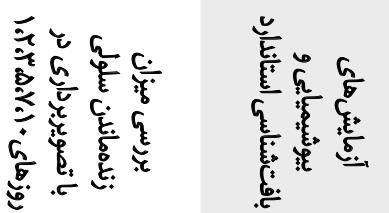

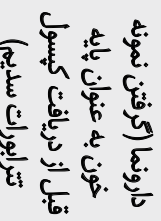

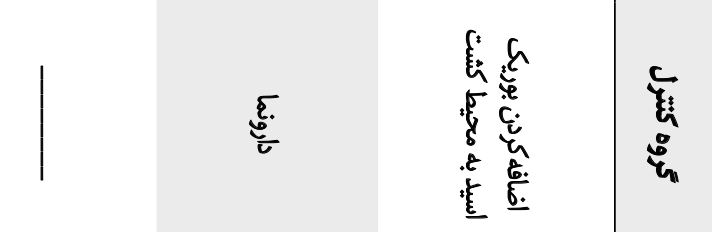

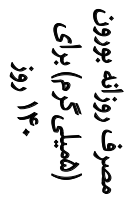
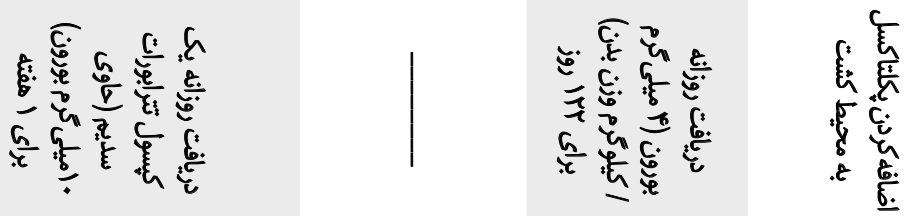

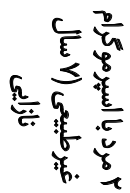

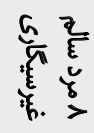

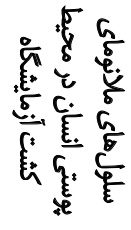

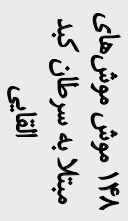

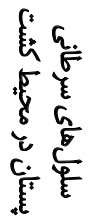

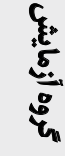

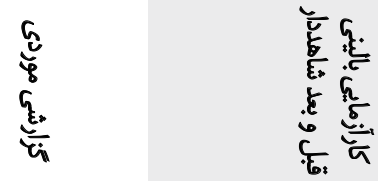

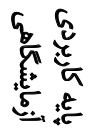

$\frac{a}{c^{2}}$

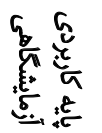

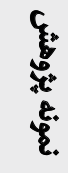

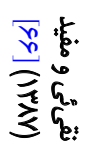

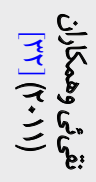

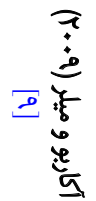

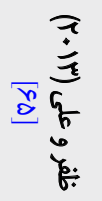

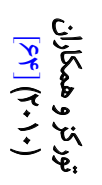

द्.

参

$\frac{5}{6}$

है

$\hbar$
$\hbar$
$c$
$c$
$k$

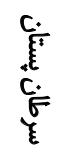

$\frac{E}{E}$

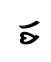

天

๕

$=$

s. 
سلامتى در نظر ترفته شود و افزايش مصرف بورون با رئيمهاى

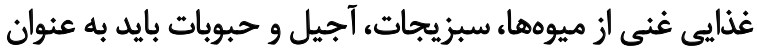

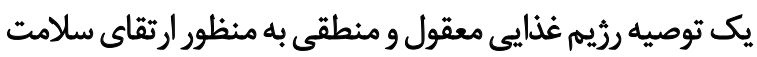

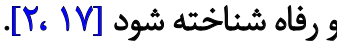
از جمله محدوديتهاي اين مطالعه مقالاتى بهزبان غيرانكليسى

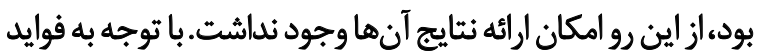

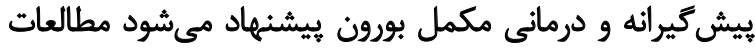

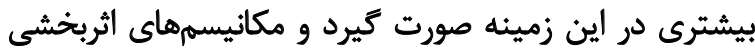

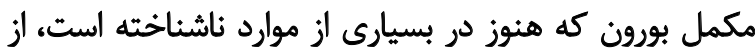

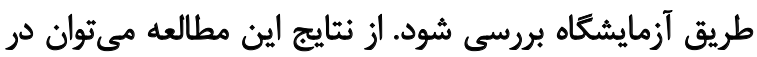

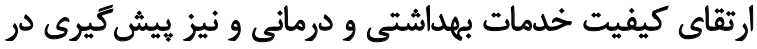

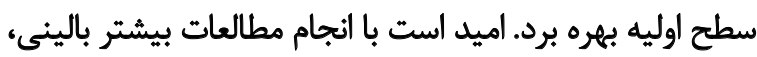

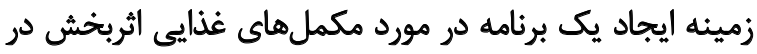

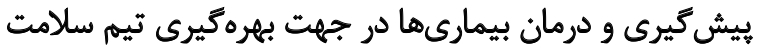

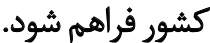

\section{تيجيليرى}

مكمل بورون عملكرد مثبتى براى رشد استخوانها و سيستم

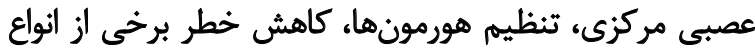

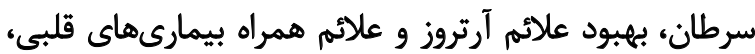

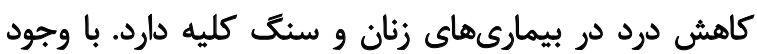

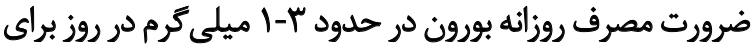

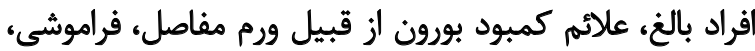

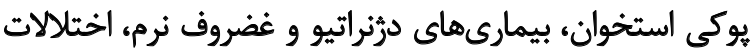

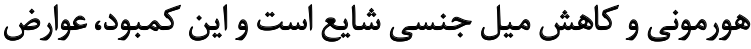

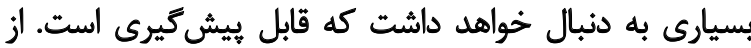

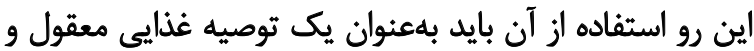
جانشينى مناسب براى داروهاى شيميايى رايج توصيه شود.

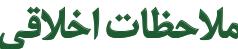 يبيروى از اصول اخلاق يثوهش}

مقاله به صورت مرورى بر اساس بررسى ساير متون و مقالات نوشته شده است و شركت كنينده و كداخلاقي نداشته است.

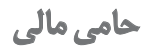

ايناين مقاله مرورى منظم، حامى مالى نداشته است و بركرفته از

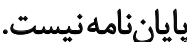
مشاركت ثويسند

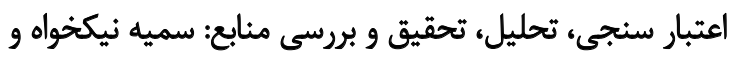

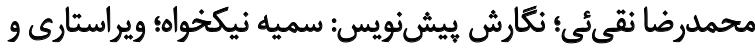

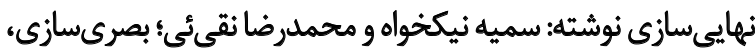

آرتروز و بيمارىهاى مرتبط با اندوتوكسينها ايفا كنند [IT، TV]. با وجود برخى تحقيقات معتقدند كه بورون در تعديل آبشار

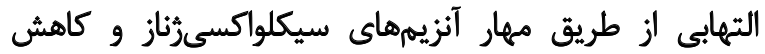

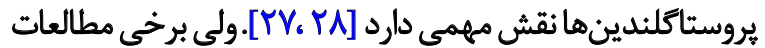

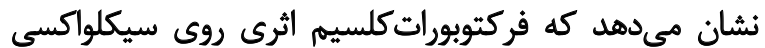

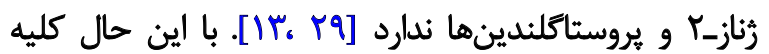

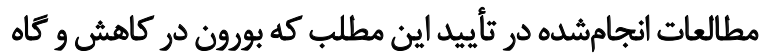

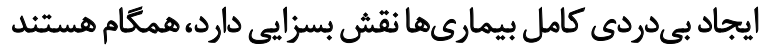

[1 $19 . Y \cdot 6 r-Y \Delta 0]$

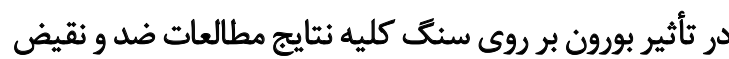

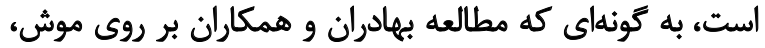

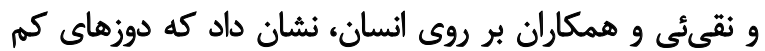

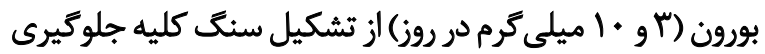

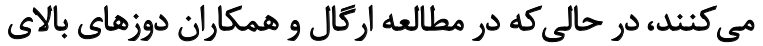

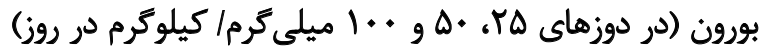

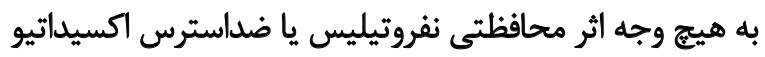

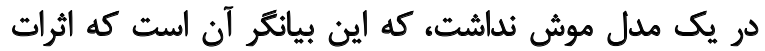

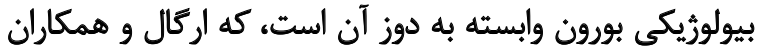

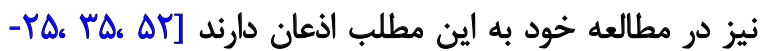

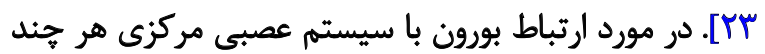

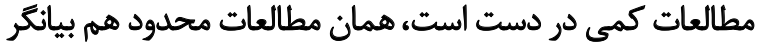

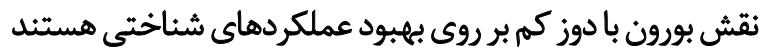

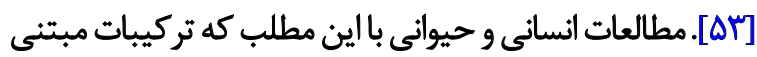

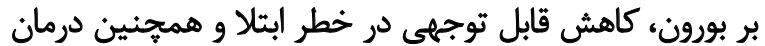

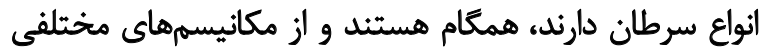

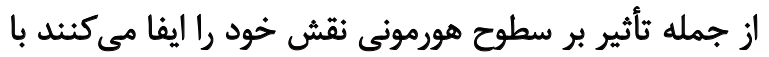

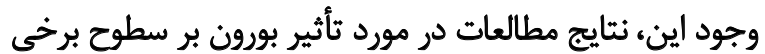

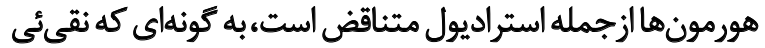

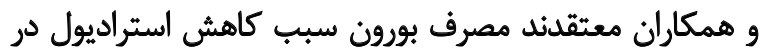

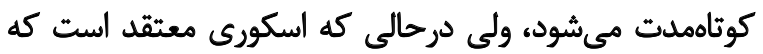

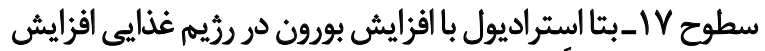

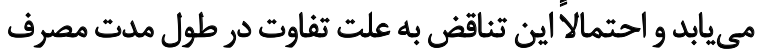

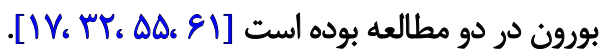

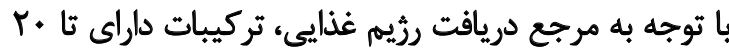

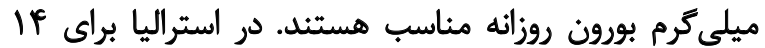

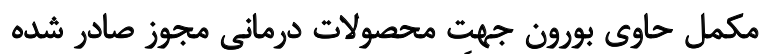

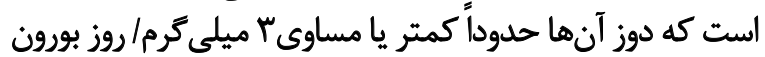

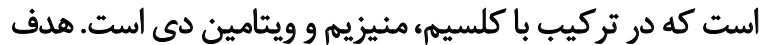

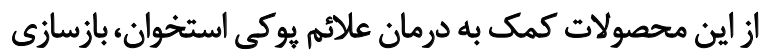

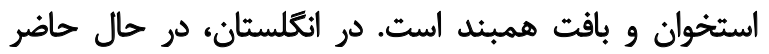

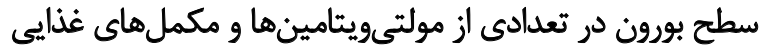

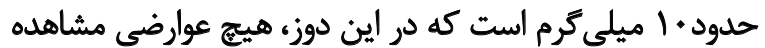

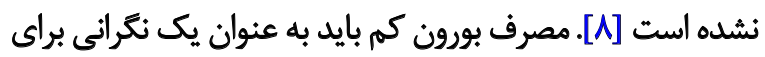




$$
\text { تظارت، مديريت يرورٌه و تأمين مالى: سميه نيكخواه. }
$$

بنابر اظهار نويسندكان اين مقاله تعارض منافع ندارد.

$$
\text { تشكر و قدردانى }
$$

بدينوسيله از همكارى يرفسور نيلسن و هانت كه از طريق

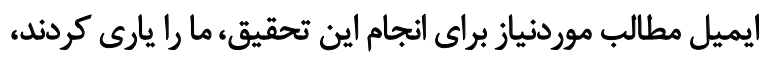
كمال تشكر و قدردانى را داريم. 


\section{References}

[1] Poddar P, Hidell H, Agro CE, Collete M. Trivalent chromium and boron fortifying composition, a hydration supplement, and process for preparing the same [Internet]. 2011 [Updated 2011 September 23]. Available from: https://patents.google.com/patent/ WO2012038985A3/und

[2] Nielsen FH, Meacham SL. Growing evidence for human health benefits ofboron.Journal of Evidence-Based Complementary\&Alternative Medicine. 2011; 16(3):169-80. [DOI:10.1177/2156587211407638]

[3] Warington $\mathrm{K}$. The effect of boric acid and borax on the broad bean and certain other plants. Annals of Botany. 1923; 37(4):629-72. [DOI:10.1093/oxfordjournals.aob.a089871]

[4] Catharine Ross A, editor. Modern nutrition in health and disease. 11th ed. Philadelphia: Wolters Kluwer Health/Lippincott Williams \& Wilkins; 2014

[5] Hosmane NS, editor. Boron science: New technologies and applications. Boca Raton: CRC Press; 2012.

[6] Ciani L, Ristori S. Boron as a platform for new drug design. Expert Opinion on Drug Discovery. 2012; 7(11):1017-27. [DOI:10.1517/174 60441.2012.717530] [PMID]

[7] Scorei IR. Boron compounds in the breast cancer cells chemoprevention and chemotherapy. In: Gunduz E, Gunduz M, editors. Breast Cancer - Current and Alternative Therapeutic Modalities. Rijeka: IntechOpen; 2011.

[8] Dinca L, Scorei R. Boron in human nutrition and its regulations use. Journal of Nutritional Therapeutics. 2013; 2(1):22-9.

[9] Acerbo AS, Miller LM. Assessment of the chemical changes induced in human melanoma cells by boric acid treatment using infrared imaging. Analyst. 2009; 134(8):1669-74. [DOI:10.1039/b823234b] [PMID]

[10] Hunt CD. Dietary boron: Progress in establishing essential roles in human physiology. Journal of Trace Elements in Medicine and Biology. 2012; 26(2):157-60. [DOI:10.1016/j.jtemb.2012.03.014] [PMID]

[11] Nielsen FH. Manganese, molybdenum, boron, chromium, and other trace elements. In: Erdman Jr JW, Macdonald IA, Zeisel SH, editors. Present Knowledge in Nutrition. 10th ed. Washington D.C: International Life Sciences Institute; 2012. [DOI:10.1002/9781119946045. ch38]

[12] Liao SF, Monegue JS, Lindemann MD, Cromwell GL, Matthews JC. Dietary supplementation of boron differentially alters expression of borate transporter $(\mathrm{NaBCl})$ mRNA by jejunum and kidney of growing pigs. Biological Trace Element Research. 2011; 143(2):901-12. [DOI:10.1007/s12011-010-8936-2] [PMID]

[13] Scorei RI, Rotaru P. Calcium fructoborate-potential anti-inflammatory agent. Biological Trace Element Research. 2011; 143(3):1223-38. [DOI:10.1007/s12011-011-8972-6] [PMID]

[14] Meacham S, Karakas S, Wallace A, Altun F. Boron in human health: Evidence for dietary recommendations and public policies. The Open Mineral Processing Journal. 2010; 3:36-53. [DOI:10.2174/187 4841401003010036]

[15] Kathleen Mahan L, Raymond JL. Krause's food \& the nutrition care process. 14th ed. Amsterdam: Elsevier Health Sciences; 2016.

[16] Naghii M, Wall P, Samman S. The boron content of selected foods and the estimation of its daily intake among free-living subjects.
Journal of the American College of Nutrition. 1996; 15(6):614-9. [DO I:10.1080/07315724.1996.10718638] [PMID]

[17] Nielsen FH. Update on human health effects of boron. Journal of Trace Elements in Medicine and Biology. 2014; 28(4):383-7. [DOI:10.1016/j.jtemb.2014.06.023] [PMID]

[18] Naghii MR, Samman S. The role of boron in nutrition and metabolism. Progress in Food \& Nutrition Science. 1993; 17(4):331-49. [PMID]

[19] Militaru C, Donoiu I, Craciun A, Scorei ID, Bulearca AM, Scorei RI. Oral resveratrol and calcium fructoborate supplementation in subjects with stable angina pectoris: Effects on lipid profiles, inflammation markers, and quality of life. Nutrition. 2013; 29(1):178-83. [DOI:10.1016/j.nut.2012.07.006] [PMID]

[20] Reyes-Izquierdo T, Nemzer B, Gonzalez AE, Zhou Q, Argumedo $\mathrm{R}$, Shu $\mathrm{C}$, et al. Short-term intake of calcium fructoborate improves WOMAC and McGill scores and beneficially modulates biomarkers associated with knee osteoarthritis: A pilot clinical double-blinded placebo-controlled study. American Journal of Biomedical Sciences. 2012; 4(2):111-22. [DOI:10.5099/aj120200111]

[21] Khaliq H, Juming Z, Ke-Mei P. The physiological role of boron on health. Biological Trace Element Research. 2018; 186(1):31-51. [DOI:10.1007/s12011-018-1284-3] [PMID]

[22] Nikkhah S, Dolatian M, Naghii MR, Zaeri F, Taheri SM. Effects of boron supplementation on the severity and duration of pain in primary dysmenorrhea. Complementary Therapies in Clinical Practice. 2015 21(2):79-83. [DOI:10.1016/j.ctcp.2015.03.005] [PMID]

[23] Naghii MR, Einollahi B, Rostami Z. Preliminary evidence hints at a protective role for boron in urolithiasis. The Journal of Alternative and Complementary Medicine. 2012; 18(3):207-9. [DOI:10.1089/ acm.2011.0865] [PMID]

[24] Naghii MR. Significant pain alleviation, cease of hematuria, and renal stone removal after extracorporeal shock wave lithotripsy with adjuvant boron therapy-case report. Endocrine Regulations. 2013; 47(2):101-4. [DOI:10.4149/endo_2013_02_101] [PMID]

[25] Naghii MR. Boron and antioxidants complex: A new concept for the treatment of kidney stones without rigorous pain. Endocrine Regulations. 2014; 48(3):120-5. [DOI:10.4149/endo_2014 03 120] [PMID]

[26] Scorei IR. Calcium fructoborate: Plant-based dietary boron as potential medicine for cancer therapy. Frontiers in Bioscience. 2011 S3:205-15. [DOI:10.2741/s145] [PMID]

[27] Scorei ID, Scorei RI. Calcium fructoborate helps control inflammation associated with diminished bone health. Biological Trace Element Research. 2013; 155(3):315-21. [DOI:10.1007/s12011-013 9800-y] [PMID]

[28] Hunt CD, Idso JP. Dietary boron as a physiological regulator of the normal inflammatory response: A review and current research progress. The Journal of Trace Elements in Experimental Medicine. 1999; 12(3):221-33. [DOI:10.1002/(SICI)1520-670X(1999)12:3<221::AID JTRA6>3.0.CO;2-X]

[29] Scorei RI, Ciofrangeanu C, Ion R, Cimpean A, Galateanu B, Mitran $V$, et al. In vitro effects of calcium fructoborate upon production of inflammatory mediators by LPS-stimulated RAW 264.7 macrophages. Biological Trace Element Research. 2010; 135(1-3):334-44 [DOI:10.1007/s12011-009-8488-5] [PMID]

[30] Scorei R, Mitrut P, Petrisor I, Scorei I. A double-blind, placebo-controlled pilot study to evaluate the effect of calcium fructoborate on 
systemic inflammation and dyslipidemia markers for middle-aged people with primary osteoarthritis. Biological Trace Element Research. 2011; 144(1-3):253-63. [DOI:10.1007/s12011-011-9083-0] [PMID] [PMCID]

[31] Spears JW, Armstrong TA. Dietary boron: Evidence for a role in immune function. In: Xu F, Goldbach HE, Brown PH, Bell RW, Fujiwara T, Hunt $\mathrm{CD}$, et al, editors. Advances in plant and animal boron nutrition. Dordrecht: Springer; 2007. [DOI:10.1007/978-1-4020-5382-5_26]

[32] Naghii MR, Mofid M, Asgari AR, Hedayati M, Daneshpour MS. Comparative effects of daily and weekly boron supplementation on plasma steroid hormones and proinflammatory cytokines. Journal of Trace Elements in Medicine and Biology. 2011; 25(1):54-8. [DOI:10.1016/j.jtemb.2010.10.001] [PMID]

[33] Jadad AR, Andrew Moore R, Carroll D, Jenkinson CM, Reynolds DJM, Gavaghan DJ, et al. Assessing the quality of reports of randomized clinical trials: Is blinding necessary? Controlled Clinical Trials. 1996; 17(1):1-12. [DOI:10.1016/0197-2456(95)00134-4]

[34] Yin C, Jia X, Miron RJ, Long Q, Xu H, Wei Y, et al. Setd7 and its contribution to Boron-induced bone regeneration in Boron-mesoporous bioactive glass scaffolds. Acta Biomaterialia. 2018; 73:522-30. [DOI:10.1016/j.actbio.2018.04.033] [PMID]

[35] Ergul AB, Kara M, Karakukcu C, Tasdemir A, Aslaner H, Ergul MA, et al. High doses of boron have no protective effect against nephrolithiasis or oxidative stress in a rat model. Biological Trace Element Research. 2018; 186(1):218-25. [DOI:10.1007/s12011-018-1294-1] [PMID]

[36] Beattie JH, Peace HS. The influence of a low-boron diet and boron supplementation on bone, major mineral and sex steroid metabolism in postmenopausal women. British Journal of Nutrition. 1993; 69(3):871-84. [DOI:10.1079/BJN19930087] [PMID]

[37] Nielsen FH, Hunt CD, Mullen LM, Hunt JR. Effect of dietary boron on mineral, estrogen, and testosterone metabolism in postmenopausal women. The FASEB Journal. 1987; 1(5):394-7. [DOI:10.1096/ fasebj.1.5.3678698] [PMID]

[38] Naghii M, Samman S. The effect of boron supplementation on its urinary excretion and selected cardiovascular risk factors in healthy male subjects. Biological Trace Element Research. 1997; 56(3):27386. [DOI:10.1007/BF02785299] [PMID]

[39] Ghanizadeh G, Babaei M, Naghii MR, Mofid M, Torkaman G, Hedayati $M$. The effect of supplementation of calcium, vitamin $D$, boron, and increased fluoride intake on bone mechanical properties and metabolic hormones in rat. Toxicology and Industrial Health. 2014; 30(3):211-7. [DOI:10.1177/0748233712452775] [PMID]

[40] Nielsen FH, Stoecker BJ. Boron and fish oil have different beneficial effects on strength and trabecular microarchitecture of bone. Journal of Trace Elements in Medicine and Biology. 2009; 23(3):195-203. [DOI:10.1016/j.jtemb.2009.03.003] [PMID]

[41] Gorustovich AA, Steimetz T, Nielsen FH, Guglielmotti MB. Histomorphometric study of alveolar bone healing in rats fed a boron-deficient diet. The Anatomical Record. 2009; 291(4):441-7. [DOI:10.1002/ar.20672] [PMID]

[42] Armstrong TA, Spears JW, Crenshaw TD, Nielsen FH. Boron supplementation of a semipurified diet for weanling pigs improves feed efficiency and bone strength characteristics and alters plasma lipid metabolites. The Journal of Nutrition. 2000; 130(10):2575-81. [DOI:10.1093/jn/130.10.2575] [PMID]

[43] Armstrong T, Spears J, Lloyd K. Inflammatory response, growth, and thyroid hormone concentrations are affected by long-term boron supplementation in gilts. Journal of Animal Science. 2001 79(6):1549-56. [DOI:10.2527/2001.7961549x] [PMID]

[44] Taranu I, Marin DE, Manda G, Motiu M, Neagoe I, Tabuc C, et al. Assessment of the potential of a boron-fructose additive in counteracting the toxic effect of Fusarium mycotoxins. British Journal of Nutrition. 2011; 106(3):398-407. [DOI:10.1017/S0007114511000341] [PMID]

[45] Hunt CD. Dietary boron: Evidence for essentiality and homeostatic control in humans and animals. In: Xu F, Goldbach HE, Brown PH, Bell RW, Fujiwara T, Hunt CD, et al, editors. Advances in plant and animal Boron nutrition. Dordrecht: Springer; 2007. [DOI:10.1007/978-14020-5382-5_25]

[46] Samman S, Naghii MR, Lyons Wall PM, Verus AP. The nutritiona and metabolic effects of boron in humans and animals. Biological Trace Element Research. 1998; 66(1-3):227-35. [DOI:10.1007/ BF02783140] [PMID]

[47] Nguyen AM, Humphrey L, Kitchen H, Rehman T, Norquist JM. A qualitative study to develop a patient-reported outcome for dysmenorrhea. Quality of Life Research. 2014; 24(1):181-91. [DOI:10.1007/ s11136-014-0755-z] [PMID]

[48] Ayan M, Sogut E, Tas U, Erdemir F, Sahin M, Suren M, et al. Pain levels associated with renal colic and primary dysmenorrhea: A prospective controlled study with objective and subjective outcomes. Archives of Gynecology and Obstetrics. 2012; 286(2):403-9. [DOI:10.1007/s00404-012-2316-4] [PMID]

[49] Fritz MA, Speroff L. Clinical gynecologic endocrinology and infertility. Philadelphia: Lippincott Williams \& Wilkins; 2012.

[50] Park JS, Park S, Cheon CH, Go HY, Sun SH, Shin YC, et al. Effects of Gyejibongnyeong-hwan on dysmenorrhea caused by blood stagnation: Study protocol for a randomized controlled trial. Trials. 2012 13:3. [DOI:10.1186/1745-6215-13-3] [PMID] [PMCID]

[51] Khan SR. Reactive oxygen species as the molecular modulators of calcium oxalate kidney stone formation: Evidence from clinical and experimental investigations. The Journal of Urology. 2013 189(3):803-11. [DOI:10.1016/j.juro.2012.05.078] [PMID] [PMCID]

[52] Bahadoran H, Naghii MR, Mofid M, Asadi MH, Ahmadi K, Sarveazad A. Protective effects of boron and vitamin E on ethylene glycol-induced renal crystal calcium deposition in rat. Endocrine Regulations. 2016; 50(4):194-206. [DOI:10.1515/enr-2016-0021] [PMID]

[53] Nielsen FH, Penland JG. Boron deprivation alters rat behaviour and brain mineral composition differently when fish oil instead of safflower oil is the diet fat source. Nutritional Neuroscience. 2006; 9(1-2):105-12. [DOI:10.1080/10284150600772189] [PMID]

[54] Alak G, Parlak V, Aslan ME, Ucar A, Atamanalp M, Turkez H. Borax supplementation alleviates hematotoxicity and DNA damage in rainbow trout (Oncorhynchus mykiss) exposed to copper. Biological Trace Element Research. 2018; 187(2):536-42. [DOI:10.1007/ s12011-018-1399-6] [PMID]

[55] Scorei RI, Popa R Jr. Boron-containing compounds as preventive and chemotherapeutic agents for cancer. Anti-Cancer Agents in Medicinal Chemistry (Formerly current medicinal chemistry - AntiCancer agents). 2010; 10(4):346-51. [DOI:10.2174/1871520107911 62289] [PMID]

[56] Gallardo-Williams MT, Chapin RE, King PE, Moser GJ, Goldsworthy $\mathrm{TL}$, Morrison JP, et al. Boron supplementation inhibits the growth and local expression of IGF-1 in human prostate adenocarcinoma (LNCaP) tumors in nude mice. Toxicologic Pathology. 2004; 32(1):73-8. [DOI:10.1080/01926230490260899] [PMID] 
[57] Müezzinoğlu T, Korkmaz M, Neşe N, Bakırdere S, Arslan Y, Ataman OY, et al. Prevalence of prostate cancer in high boron-exposed population: A community-based study. Biological Trace Element Research. 2011; 144(1-3):49-57. [DOI:10.1007/s12011-011-9023-z] [PMID]

[58] Scorei RI, Popa R. Sugar-borate esters-potential chemical agents in prostate cancer chemoprevention. Anti-Cancer Agents in Medicinal Chemistry (Formerly current medicinal chemistry - Anti-Cancer agents). 2013; 13(6):901-9. [DOI:10.2174/18715206113139990124 ] [PMID]

[59] Korkmaz M, Uzgören E, Bakirdere S, Aydin F, Ataman OY. Effects of dietary boron on cervical cytopathology and on micronucleus frequency in exfoliated buccal cells. Environmental Toxicology. 2007; 22(1):17-25. [DOI:10.1002/tox.20229] [PMID]

[60] Mahabir S, Spitz MR, Barrera SL, Dong YQ, Eastham C, Forman MR. Dietary boron and hormone replacement therapy as risk factors for lung cancer in women. American Journal of Epidemiology. 2008; 167(9):1070-80. [DOI:10.1093/aje/kwn021] [PMID] [PMCID]

[61] Scorei R. Is boron a prebiotic element? A mini-review of the essentiality of boron for the appearance of life on earth. Origins of Life and Evolution of Biospheres. 2012; 42(1):3-17. [DOI:10.1007/s11084012-9269-2] [PMID]

[62] Hofling M, Hirschberg AL, Skoog L, Tani E, Hägerström T, von Schoultz B. Testosterone inhibits estrogen/progestogen-induced breast cell proliferation in postmenopausal women. Menopause. 2007; 14(2):183-90. [DOI:10.1097/01.gme.0000232033.92411.51] [PMID]

[63] Jiang Q, Zhong Q, Zhang Q, Zheng S, Wang G. Boron-based 4-hydroxytamoxifen bioisosteres for treatment of de novo tamoxifen resistant breast cancer. ACS Medicinal Chemistry Letters. 2012; 3(5):392-6. [DOI:10.1021/ml3000287] [PMID] [PMCID]

[64] Turkez H, Tatar A, Hacimuftuoglu A, Ozdemir E. Boric acid as a protector against paclitaxel genotoxicity. Acta Biochimica Polonica. 2010; 57(1):95-7. [DOI:10.18388/abp.2010_2378] [PMID]

[65] Zafar H, Ali Sh. Boron inhibits the proliferating cell nuclear antigen index, molybdenum containing proteins and ameliorates oxidative stress in hepatocellular carcinoma. Archives of Biochemistry and Biophysics. 2013; 529(2):66-74. [DOI:10.1016/j.abb.2012.11.008] [PMID]

[66] Naghii MR, Mofid M. [Boron effect on sperm parameters in two infertile individuals, case report (Persian)]. Kowsar Medical Journal. 2008; 13(1):79-82.

[67] Fort DJ, Propst TL, Stover EL, Strong PL, Murray FJ. Adverse reproductive and developmental effects in Xenopus from insufficient boron. Biological Trace Element Research. 1998; 66(1-3):237-59. [DOI:10.1007/BF02783141] [PMID]

[68] Eckhert CD. Boron stimulates embryonic trout growth. The Journal of Nutrition. 1998; 128(12):2488-93. [DOI:10.1093/jn/128.12.2488] [PMID]

[69] Rowe RI, Eckhert CD. Boron is required for zebrafish embryogenesis. Journal of Experimental Biology. 1999; 202(Pt 12):1649-54. [PMID]

[70] Baker SJ, Tomsho JW, Benkovic SJ. Boron-containing inhibitors of synthetases. Chemical Society Reviews. 2011; 40(8):4279-85. [DOI:10.1039/c0cs00131g] [PMID]

[71] Dembitsky VM, Al Quntar AA, Srebnik M. Natural and synthetic small boron-containing molecules as potential inhibitors of bacterial and fungal quorum sensing. Chemical Reviews. 2010; 111(1):209-37. [DOI:10.1021/cr100093b] [PMID]
[72] Nieves J. Skeletal effects of nutrients and nutraceuticals, beyond calcium and vitamin D. Osteoporosis International. 2013; 24(3):77186. [DOI:10.1007/s00198-012-2214-4] [PMID]

[73] Bailey RL, Gahche JJ, Miller PE, Thomas PR, Dwyer JT. Why US adults use dietary supplements. JAMA Internal Medicine. 2013; 173(5):355-61. [DOI:10.1001/jamainternmed.2013.2299] [PMID]

[74] Kurtoğlu F, Kurtoğlu V, Çelik I, Keçeci T, Nizamlioğlu M. Effects of dietary boron supplementation on some biochemical parameters, peripheral blood lymphocytes, splenic plasma cells and bone characteristics of broiler chicks given diets with adequate or inadequate cholecalciferol (vitamin D3) content. British Poultry Science. 2005; 46(1):87-96. [DOI:10.1080/00071660400024001] [PMID] 